\title{
NEW YOUNG STAR CANDIDATES IN THE TAURUS-AURIGA REGION AS SELECTED FROM THE WIDE-FIELD INFRARED SURVEY EXPLORER
}

\author{
L. M. Rebull ${ }^{1}$, X. P. Koenig ${ }^{2,8}$, D. L. PadgetT ${ }^{1,9}$, S. Terebey ${ }^{3}$, P. M. McGehee ${ }^{4}$, L. A. Hillenbrand ${ }^{5}$, G. R. KnapP ${ }^{6}$,

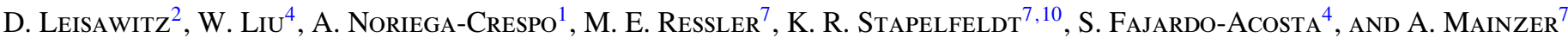 \\ ${ }^{1}$ Spitzer Science Center (SSC), California Institute of Technology, M/S 220-6, 1200 E. California Blvd., Pasadena, CA 91125, USA; \\ rebull@ipac.caltech.edu, alberto@ipac.caltech.edu \\ ${ }^{2}$ NASA Goddard Space Flight Center (GSFC), M/S 605, Greenbelt, MD 20771, USA; xavier.p.koenig@ nasa.gov, david.t.leisawitz@nasa.gov \\ ${ }^{3}$ Department of Physics and Astronomy, California State University at Los Angeles, 5151 State University Dr., Los Angeles, CA 90032, USA; sterebe@ calstatela.edu \\ ${ }^{4}$ Infrared Processing and Analysis Center (IPAC), California Institute of Technology, 1200 E. California Blvd., Pasadena, CA 91125, USA; \\ peregrin@ipac.caltech.edu,wliu@ipac.caltech.edu,fajardo@ipac.caltech.edu \\ ${ }^{5}$ California Institute of Technology, M/S 249-17, 1200 E. California Blvd., Pasadena, CA 91125, USA; lah@ astro.caltech.edu \\ ${ }^{6}$ Department of Astrophysical Sciences, Princeton University, Princeton, NJ 08544, USA; gk @ astro.princeton.edu \\ ${ }^{7}$ Jet Propulsion Laboratory, California Institute of Technology, 4800 Oak Grove Drive, Pasadena, CA 91109, USA; \\ michael.e.ressler@jpl.nasa.gov, amainzer@jpl.nasa.gov \\ Received 2011 April 28; accepted 2011 June 24; published 2011 August 19
}

\begin{abstract}
The Taurus Molecular Cloud subtends a large solid angle on the sky, in excess of $250 \mathrm{deg}^{2}$. The search for legitimate Taurus members to date has been limited by sky coverage as well as the challenge of distinguishing members from field interlopers. The Wide-field Infrared Survey Explorer has recently observed the entire sky, and we take advantage of the opportunity to search for young stellar object (YSO) candidate Taurus members from a $\sim 260 \mathrm{deg}^{2}$ region designed to encompass previously identified Taurus members. We use near- and mid-infrared colors to select objects with apparent infrared excesses and incorporate other catalogs of ancillary data to present a list of rediscovered Taurus YSOs with infrared excesses (taken to be due to circumstellar disks), a list of rejected YSO candidates (largely galaxies), and a list of 94 surviving candidate new YSO-like Taurus members. There is likely to be contamination lingering in this candidate list, and follow-up spectra are warranted.
\end{abstract}

Key words: circumstellar matter - infrared: stars - stars: formation - stars: pre-main sequence

Online-only material: color figures, figure set, machine-readable tables

\section{INTRODUCTION}

In recent years, there have been many infrared (IR) studies of nearby star-forming regions with the Spitzer Space Telescope (Werner et al. 2004). Since most, if not all, low-mass stars form with circumstellar accretion disks, they have IR excesses for as long as the dusty circumstellar material survives (e.g., Hernandez et al. 2008). Stars with large IR excesses are relatively easily distinguished from stars without such excesses, while stars with more modest excesses require more accurate knowledge of the underlying star and the intervening extinction. Surveys with Spitzer have proven very good at finding new young stars in star-forming regions, some located surprisingly far from the traditional locations of star formation based on $\mathrm{CO}$ gas or IRAS dust maps. However, even though Spitzer is able to survey large areas relatively quickly, it still is a pointed observatory and cannot easily conduct a truly wide-field survey. In very close star-forming regions such as Taurus, the large solid angle subtended by the association challenges the search for new members, rendering the samples incomplete, particularly for a distributed population. See, however, Rebull et al. (2010).

We conducted a $\sim 44 \mathrm{deg}^{2}$ survey of Taurus with Spitzer, using 3.6, 4.5, 5.8, 8, 24, 70, and $160 \mu \mathrm{m}$ (D. L. Padgett et al. 2011, in preparation; Padgett et al. 2008b; Güdel et al. 2007b). In Rebull et al. (2010), we reported on our search for new young stellar objects (YSOs) in Taurus. We used a primarily Spitzer-driven

\footnotetext{
8 NASA Postdoctoral Program Fellow.

9 Currently at NASA Goddard Space Flight Center (GSFC), Code 665, Greenbelt, MD 20771, USA; Deborah.L.Padgett@nasa.gov.

${ }^{10}$ Currently at NASA Goddard Space Flight Center (GSFC), Code 667, Greenbelt, MD 20771, USA; karl.r.stapelfeldt@ nasa.gov.
}

color selection, but took advantage of considerable ancillary data amassed in the service of a multi-wavelength search for new young stars. We found that any solely near- and midinfrared color selection was filled with contamination from galaxies and asymptotic giant branch (AGB) stars, and that use of ancillary data was crucial to establishing a list of highquality new members of Taurus. We now use our experience with this prior data set to inform our selection using the Wide-field Infrared Survey Explorer (WISE; Wright et al. 2010) data.

WISE conducted an all-sky survey in 2009-2011. These data are well-suited to studying nearby star-forming clouds such as Taurus, where members are likely to be bright because of their proximity to us as well as their youth, and well within the regime of good signal-to-noise ratio $(\mathrm{S} / \mathrm{N})$ WISE photometry. Furthermore, the all-sky nature of the survey allows us to look away from the more traditional and well-studied clustered regions and include some young stars in the direction of Taurus (not necessarily Taurus members) that were never studied with Spitzer.

WISE surveyed the entire sky, but the depth of coverage is nonuniform and generally greater at higher ecliptic latitudes (Taurus is in the ecliptic plane). The depth of coverage in the Taurus region is somewhat degraded relative to regions of comparable ecliptic latitude due to Moon avoidance maneuvers made during the mission. Most of the Taurus region is observed to a depth of about $0.08,0.11,0.8$, and $6 \mathrm{mJy}$ in the four bands $(5 \sigma$, given eight visits), and we further restrict the depth with $\mathrm{S} / \mathrm{N}$ cuts; see below. Our original Taurus Spitzer Survey went to 0.06, $0.06,0.14$, and $1.1 \mathrm{mJy}$ for IRAC-1 $(3.6 \mu \mathrm{m})$, IRAC-2 $(4.5 \mu \mathrm{m})$, IRAC-4 ( $8 \mu \mathrm{m})$, and MIPS-24 $(24 \mu \mathrm{m})$, respectively. However, both surveys should easily detect legitimate Taurus members, since the cloud is only $140 \mathrm{pc}$ away. 


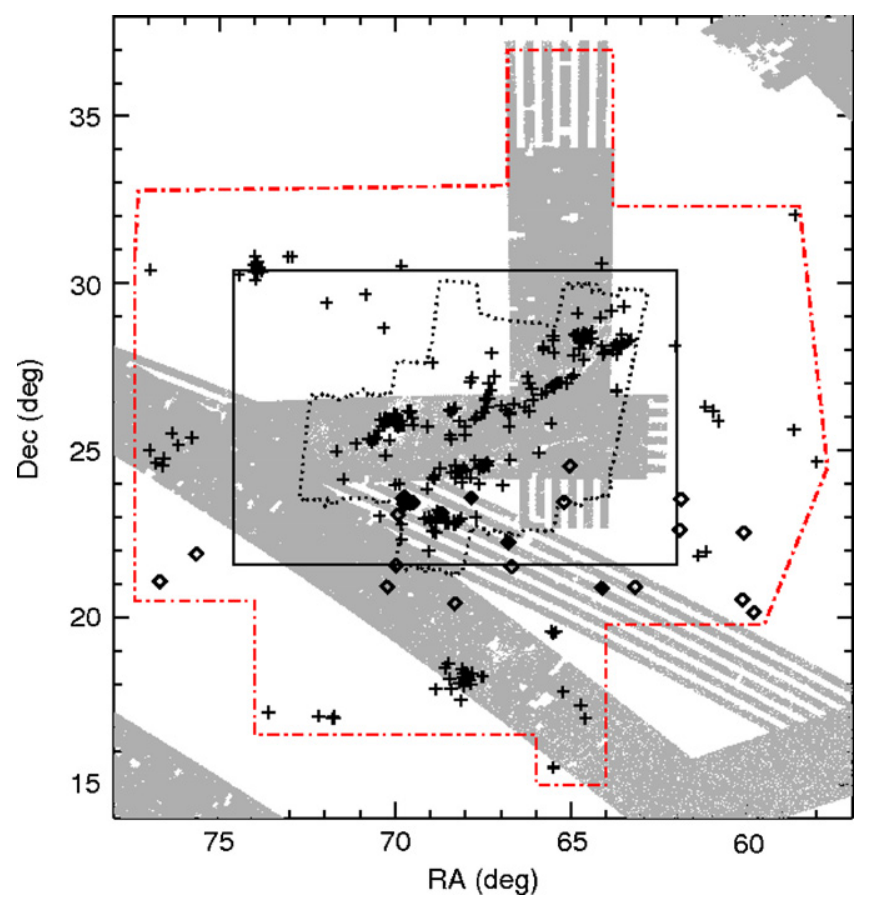

Figure 1. Location in the sky of the various surveys discussed here. Solid black box: boundary of Goldsmith et al. (2008) CO(1-0) survey; gray-colored regions: SDSS coverage; smaller irregular black dotted polygon: coverage of Spitzer Taurus Survey (D. L. Padgett et al. 2011, in preparation; Padgett et al. 2008b; Güdel et al. 2007b); red dash-dotted line: boundary of polygon extracted from WISE catalog; + symbols: Taurus members from Kenyon et al. (2008); diamonds: proposed Taurus members from Slesnick et al. (2006).

(A color version of this figure is available in the online journal.)

In this paper, we select new candidate Taurus members with infrared excesses using WISE colors using the method established in Koenig et al. (2011). We compare the list of selected objects to catalogs we have assembled from our prior work and updated. We report three lists-recovered young stars, rejected objects, and candidate new Taurus members. The observations and basic data handling are described in Section 2, Section 3 describes how we identified our YSO candidates, Section 4 presents some global properties of the ensemble of YSO candidates, with a special focus on estimating the degree of contamination, and finally we summarize in Section 5.

\section{OBSERVATIONS, DATA REDUCTION, AND ANCILLARY DATA}

In the context of Rebull et al. (2010), we assembled a substantial multi-wavelength database, spanning Sloan $u$ through Spitzer/MIPS $160 \mu \mathrm{m}$ (with some X-rays) for point sources throughout the Taurus region. We note that not every source has photometry at all bands due to variations in depth and spatial coverage among the surveys involved. We use that catalog as the core for our analysis here, updating it with confirmed Taurus members from, e.g., Kenyon et al. (2008) and Luhman et al. (2010) outside of our original Spitzer map. We have also searched SIMBAD (and literature references therein) for known galaxies and other contaminants such as planetary nebulae (PNe) in this vicinity and considered these identifications in our analysis of the WISE photometry.

Our Taurus Spitzer Survey spanned $\sim 44 \mathrm{deg}^{2}$; see Figure 1. Notably, two of the surveys we assembled at other wavelengths extend well beyond the region we mapped with Spitzer-the CO(1-0) radio map (Goldsmith et al. 2008) and two Sloan Dig- ital Sky Survey (SDSS) stripes. Goldsmith et al. (2008) mapped $\sim 100 \mathrm{deg}^{2}$; see Figure 1. The SDSS (Finkbeiner et al. 2004; Padmanabhan et al. 2008) initially mapped the Taurus region in two perpendicular strips covering $\sim 48 \mathrm{deg}^{2}$, overlapping in part with the Goldsmith et al. (2008) and our Spitzer maps, but extending further east-west and substantially further north. Additional SDSS strips substantially increased the area observed; see Figure 1. We note that the unfilled stripe that crosses the region diagonally is one of the photometric calibration stripes a la Padmanabhan et al. (2008). Further motivating consideration of a wider area, the XMM-Newton Extended Survey of the Taurus Molecular Cloud program (Güdel et al. 2007a, and references therein) mapped $\sim 5 \mathrm{deg}^{2}$, most but not all of which was covered by our Spitzer maps. Finally, as seen in Figure 1, Kenyon et al. (2008) include a list of known Taurus members, many of which are well beyond the bounds of our original Spitzer survey, and Slesnick et al. (2006) report on several more candidate Taurus members.

We define a polygon in which we searched for YSO candidates using WISE that encompasses all of the members from Kenyon et al. (2008), a few of the potential members from Slesnick et al. (2006), and all of the Sloan coverage to the north of our previous Spitzer survey. The R.A./decl. vertices of this polygon are in degrees: $77.431,77.420 .5,7420.5,7416.5,66$ 16.5, 66 15, 64 15, 64 19.8, $59.519 .8,57.7$ 24.5, 58.5 32.3, 63.8 $32.3,63.837,66.837,66.8132 .93,77.3032 .77$. The total area we have considered is $\sim 260 \mathrm{deg}^{2}$.

This area is so large that we need to consider other starforming regions very nearby (in projection) to Taurus. Figure 2 shows our polygon on an IRAS $100 \mu \mathrm{m}$ image of the region. NGC 1579 is the bright knot in the central north of the image; this is thought to be 700 pc away (Herbig et al. 2004), too far to be part of Taurus at 140 pc. Lynds 1548 and environs will be discussed in a forthcoming paper by W. Liu et al. (2012, in preparation). Our polygon intersects with the tidal radius of the Pleiades $\left(\sim 6^{\circ}\right.$; Adams et al. 2001 ). Young objects identified within our polygon and within $\sim 6^{\circ}$ of the Pleiades could belong to the Pleiades and not Taurus.

WISE data acquisition and reduction are discussed in Wright et al. (2010), Jarrett et al. (2011), and in the Explanatory Supplement to the WISE Preliminary Data Release Products. There are four WISE bands, with central wavelengths at 3.4, 4.6, 12 , and $22 \mu \mathrm{m}$, and a spatial resolution of $6^{\prime \prime}\left(12^{\prime \prime}\right.$ at $\left.22 \mu \mathrm{m}\right)$. The four bands are often referred to as W1, W2, W3, and W4. We rejected any source with contamination and confusion flags ("cc_flags" in the catalog) that include any of the characters "DHOP" in its four-character string as likely contamination or confusion artifacts. ${ }^{11}$ This process resulted in a catalog containing about 2.38 million sources. The WISE catalog reports $\mathrm{S} /$ Ns. We further restricted ourselves to those measurements with $\mathrm{S} / \mathrm{N}>7$ in all four bands, drastically shrinking the catalog to $\sim 7000$ sources. Our initial attempts limited the analysis to those measurements with $\mathrm{S} / \mathrm{N}>7$ in just the first three bands, but given the expected brightness of Taurus members, plus the contamination rate we expect based on our experience with Spitzer in Taurus, we opted to enforce the S/N cut in W4 as well so as to limit the contamination in our list of candidate YSOs.

The WISE catalog reports flux measurements in magnitudes with errors in magnitudes, and the source selection in this paper

\footnotetext{
11 " $\mathrm{D}$ " denotes diffraction spike, " $\mathrm{H}$ " indicates that the source is spurious as a result of, or contaminated by, the scattered light halo surrounding a nearby bright source, "O" denotes optical ghost, and "P" denotes persistence artifact.
} 


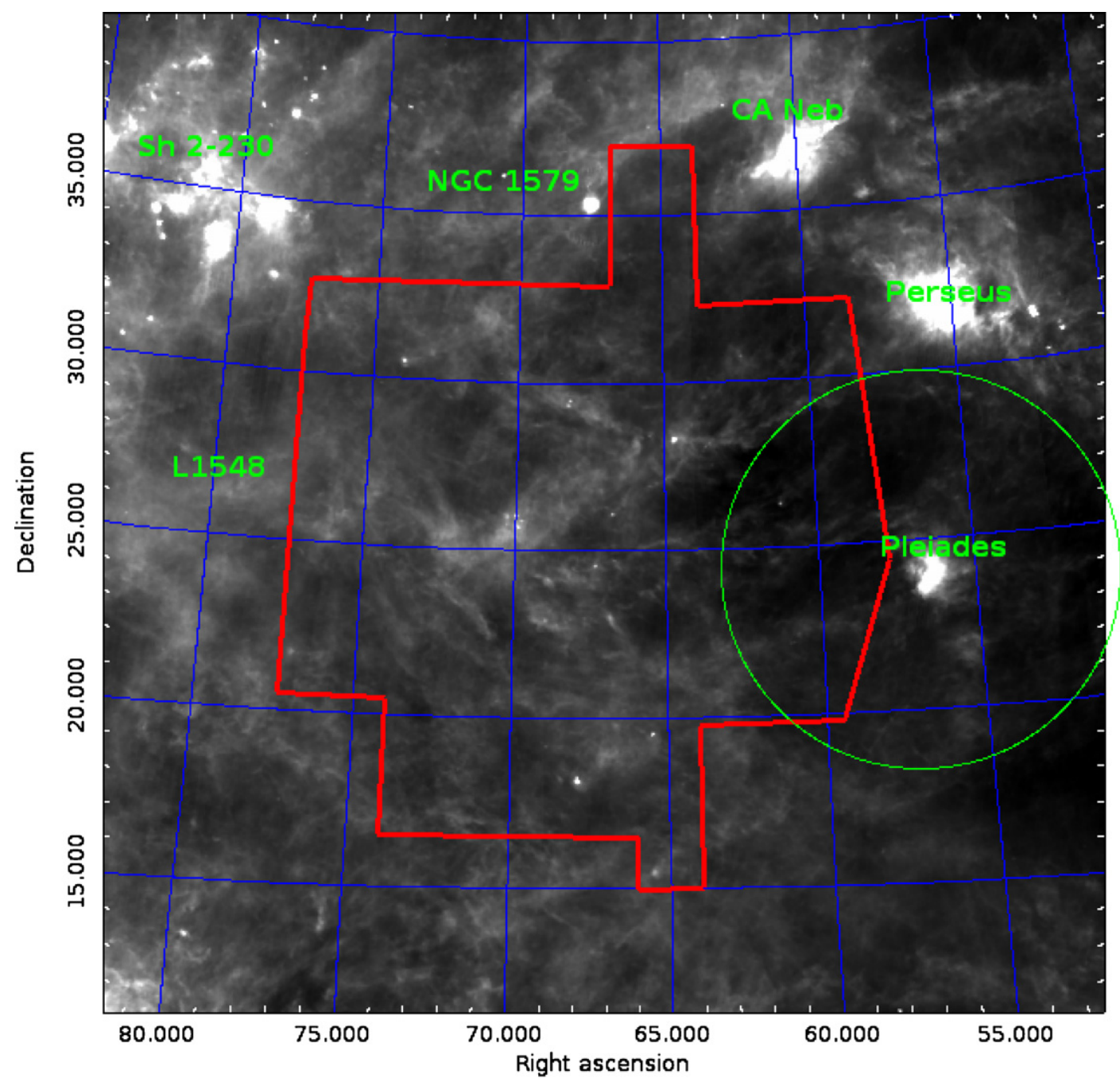

Figure 2. Overlaid on an IRAS $100 \mu \mathrm{m}$ map, the location in the sky of our survey contour (red polygon), other nearby star-forming regions of interest, and the tidal radius of the Pleiades (green circle). L1548 will be discussed by W. Liu et al. (2012, in preparation). Some of our objects are within the tidal radius of the Pleiades and thus perhaps could be members of the Pleiades rather than Taurus. NGC 1579 is centered on the bright spot.

was based on these magnitudes. The zero points we used to convert between magnitudes and flux densities in the spectral energy distribution (SED) plots included in the complete figure set of Figure 12 in the Appendix are 309.54, 171.79, 31.676, and $8.3635 \mathrm{Jy}$ for the four channels, respectively, based on the zero points in Wright et al. (2010) and the flux correction factors for a spectrum with constant $F_{v}$. These zero points are also used by Jarrett et al. (2011).

The WISE bands are merged among themselves and to the Two Micron All Sky Survey (2MASS; Skrutskie et al. 2006) in the data products provided by the WISE archive housed at the Infrared Science Archive. We merged this catalog to our alreadyassembled catalog of ancillary data via a strictly position-based search with a 1" radius. As noted in Section VI.5.f of the WISE Explanatory Supplement, the WISE catalog reports positions of sources brighter than $\mathrm{W} 1<13.0$ mag to $0.2 \operatorname{arcsec}(1 \sigma)$ relative to 2MASS positions. The positions reported for fainter sources may suffer from a systematic error up to $1^{\prime \prime}$ in declination due to a pipeline coding error.

\section{IDENTIFYING THE YSO CANDIDATES}

The process of identifying the YSO candidates is a multistep process, beginning with color cuts and progressing through ancillary data, including manual checks of SEDs and images. We now describe the process that we used.

\subsection{Initial Cut}

We started with our extracted WISE catalog over the $\sim 260 \mathrm{deg}^{2}$ polygon specified above and applied the color cuts discussed in Koenig et al. (2011). In summary, there are a series of cuts in multiple color spaces based on the location of previously identified YSOs and galaxies. These are intended statistically to weed out most contaminants and find most YSOs. No color cuts can perform this task flawlessly, though many have been discussed in the literature in the context of Spitzer observations (e.g., Allen et al. 2004; Padgett et al. 2008a; Rebull et al. 2007, 2010, 2011; Harvey et al. 2007; Gutermuth et al. 2008, 2009). As discussed in Rebull et al. (2010), especially over very large fields like Taurus, where the molecular cloud does not block out most background sources and where the survey area is big enough that the chances of obtaining more unusual objects are greater, the contamination rate for any color selection is expected to be relatively large, and ancillary data are crucial for culling the list to high-quality candidates.

The color cuts described in Koenig et al. (2011) are inspired by Gutermuth et al. (2008, 2009) and Rebull et al. (2010) and applied using WISE+2MASS colors. For their full selection 
process, Koenig et al. (2011) estimate a contamination rate for "typical" star-forming regions of about 2.4 objects resembling Class Is, 3.8 objects resembling Class IIs, and 1.8 objects resembling transition disks per square degree. At this rate, with our $\sim 260 \mathrm{deg}^{2}$ map, we expect $\sim 620, \sim 990$, and $\sim 470$, respectively, for a rough total of $\sim 2000$ contaminants per square degree. As a check, we recalculated these contamination rates for a $\sim 10 \mathrm{deg}^{2}$ patch at the north and south equatorial poles, and obtained values between $\sim 1500$ and $\sim 1600$ contaminants per square degree. However, these values are sensitive to relative depths of WISE coverage (deeper at the poles than the ecliptic), any bright extended emission in the image (present here but less so in the extragalactic fields), and natural variability in the space density of stars and galaxies.

We initially applied the Koenig et al. method to the WISE catalog where the $\mathrm{S} / \mathrm{N}$ is at least 7 in channels 1,2 , and 3, but not necessarily 4 . We thus obtained $\sim 1760$ YSO candidates. Given the contamination rates expected above, we thus anticipate a high fraction of contaminants in this list. As mentioned above, given the expected brightness of Taurus members, plus the contamination rate we expect based on our experience with Spitzer in Taurus, we chose to impose an S/N cut in W4 as well so as to limit the contamination in our list of candidate YSOs. Imposing our additional requirement that the $\mathrm{S} / \mathrm{N}$ is at least 7 in all four WISE channels results in a pool of 1014 potential YSOs.

\subsection{Ancillary Data}

We know from our Spitzer search for new members of Taurus that ancillary data are very important for weeding the contaminants out from the list of potential YSO candidates. So, in addition to our catalog above, we have included the information from ancillary data in our assembly of our final YSO candidate list in an effort to limit the contamination.

We have matched our WISE catalog to the full SDSS catalog (from DR8) in this region (which includes extended source information from the images) and the 2MASS Extended Source Catalog. Objects that are extended are likely to be (though are not guaranteed to be) galaxies; see the discussion in Rebull et al. (2010). There are also 11,500 SDSS spectra in the SDSS stripes; just 27 of the sources in our list of potential YSOs find matches with SDSS spectra.

We merged to the Akari 9 and $18 \mu \mathrm{m}$ Infrared Camera (IRC) all-sky point-source catalog (Ishihara et al. 2010). There are $\sim 3000$ sources in this region, $\sim 100$ of which match to the WISEselected sources, $\sim 80 \%$ of which are for previously known Taurus members.

Based on our experience with our Taurus Spitzer Survey, we know that the YSOs are generally though not exclusively found in regions of high $A_{V}$. Thus, we expect that an estimate of $A_{V}$ toward our new candidates in this larger region will also be useful in weeding out contaminants. Froebrich et al. (2007) report on a large, $127 \times 63 \mathrm{deg}^{2}$ extinction map based on 2MASS data. We used this map to estimate $A_{V}$ toward our list of candidates. This map is calculated to a $\sim 4^{\prime}$ resolution, and we used a $3 \times 3$ pixel $\left(6^{\prime} \times 6^{\prime}\right)$ median calculated about the position of each source to estimate the $A_{V}$.

\subsection{Previously Identified YSOs}

For the 1014 potential YSOs selected from WISE+2MASS color and magnitude cuts imposed on the 2.38 million sources with good S/N photometry, 196 of them have matches to previously identified stars with indications of youth and/or infrared excesses, in the direction of Taurus. Table 1 lists these objects and their WISE measurements. Most of the objects in Table 1 are previously identified explicitly as Taurus members in Güdel et al. (2007a) and references therein, Kenyon et al. (2008), Rebull et al. (2010), and/or Luhman et al. (2010). Eighteen of the objects in Table 1 are listed as unconfirmed candidates in Rebull et al. (2010); these are identified as such in the "notes" column of Table 1. (As discussed in Rebull et al. 2010, for these objects, we could not find unambiguous spectroscopic indications of youth-such as the $\mathrm{H} \alpha$ emission line was low or absent-so additional data are needed to confirm or refute these objects as Taurus members. These objects should not be regarded as Taurus members with the same confidence as, e.g., DG Tau, but they are not necessarily clearly field interlopers either.) The candidates from Slesnick et al. (2006) would have been similarly indicated, except they are largely not recovered, save for three objects commonly taken as Taurus members (SCHJ0429595+ $2433080=$ CFHT-20, SCHJ0438586+2336352 = J0438586+ 2336352, and SCHJ0439016+2336030 = J0439016+2336030). Eight of the objects in Table 1 are not traditionally identified as Taurus members, but we recover them as having IR excess, and they appear in the literature as having some indications of youth and with appropriate proper motions for Taurus members (2MASS J04360131+1726120, 2MASS J04324107+1809239, HD 285893, HBHA 3214-06, GZ Aur, BS Tau, IRAS 05020+2518, HO Aur). These are also indicated in Table 1 in the "notes" column.

Note that this table is not meant to be a complete list of Taurus members detected with WISE, but instead a list of all objects in the direction of Taurus, identified as young, detected in WISE with $\mathrm{S} / \mathrm{N}>7$ in all four bands and identified using the Koenig et al. (2011) method as having an IR excess. Some bona fide members of Taurus are either too faint or do not have enough color excess to be identified in this manner.

\subsection{Manual Inspection}

For the remaining 818 WISE-identified IR excess objects that have not been associated previously with young stars, we conducted a preliminary sorting into "likely contaminant" or "perhaps YSO" bins. The categorization was based on matches to objects in SIMBAD (and literature therein), matches to objects we identified as contaminants in Rebull et al. (2010), matches to the 2MASS Extended Source Catalog, and identification as extended in the SDSS pipeline. We then generated SEDs using all photometric data in our database for these objects and inspected each of them. Based on our experience, we then categorized each of the objects as still possible YSO candidates, or likely extragalactic objects. This classification is easier when there are more photometric bands; objects for which there are SDSS, 2MASS, and WISE photometry are more likely appropriately classified during this process than those without optical photometry or with just WISE photometry. Therefore, this process may have dropped viable YSO candidates similar to, e.g., MHO-1 or Haro 6-39, which have very flat SEDs (and moreover MHO-1 has just WISE photometry). Statistically the objects we have dropped on this basis are very likely to be extragalactic. Because this process is not perfect, the objects dropped as a result of SED shape are identified in the "notes" column of the tables below specifically so that follow-up optical photometry (for example) can be obtained.

After this process, there were $\sim 130$ objects which we could not yet reject as YSO candidates. For these objects, we inspected the images in all four WISE bands, and, if necessary, 2MASS, 
Table 1

Multiband Measurements of WISE-identified YSOs with Previous Indications of Youth in the Literature towards the Taurus Region

\begin{tabular}{|c|c|c|c|c|c|c|c|}
\hline $\begin{array}{l}\text { Line } \\
\text { Num. }\end{array}$ & Catalog Number & Name & $\begin{array}{l}\text { W1 }(3.4 \mu \mathrm{m}) \\
\quad(\mathrm{mag})\end{array}$ & $\begin{array}{l}\text { W2 }(4.6 \mu \mathrm{m}) \\
\quad(\mathrm{mag})\end{array}$ & $\begin{array}{l}\text { W3 }(12 \mu \mathrm{m}) \\
\quad(\mathrm{mag})\end{array}$ & $\begin{array}{l}\text { W4 }(22 \mu \mathrm{m}) \\
\quad(\mathrm{mag})\end{array}$ & Notes \\
\hline 1 & J040443.06+261856.3 & IRAS $04016+26102$ & $7.67 \pm 0.03$ & $5.16 \pm 0.03$ & $2.91 \pm 0.02$ & $0.01 \pm 0.02$ & \\
\hline 2 & J041339.46+292113.8 & None & $12.78 \pm 0.03$ & $11.74 \pm 0.02$ & $9.43 \pm 0.04$ & $7.40 \pm 0.11$ & Still a candidate $\mathrm{YSO}^{\mathrm{b}}$ \\
\hline 3 & J041353.28+281123.1 & IRAS $04108+2803 \mathrm{~A}$ & $9.44 \pm 0.02$ & $8.28 \pm 0.02$ & $5.47 \pm 0.02$ & $3.60 \pm 0.02$ & \\
\hline 4 & J041354.70+281132.6 & IRAS 04108+2803 B & $9.44 \pm 0.02$ & $7.36 \pm 0.02$ & $4.33 \pm 0.02$ & $1.46 \pm 0.01$ & \\
\hline 5 & J041357.37+291819.1 & IRAS 04108+2910 & $8.00 \pm 0.02$ & $6.98 \pm 0.02$ & $4.96 \pm 0.02$ & $2.97 \pm 0.02$ & \\
\hline 6 & J041412.29+280837.1 & IRAS $04111+2800 G$ & $13.86 \pm 0.03$ & $11.73 \pm 0.03$ & $8.97 \pm 0.03$ & $4.03 \pm 0.02$ & \\
\hline 7 & J041412.91+281212.3 & V773 Tau ABC & $6.64 \pm 0.02$ & $5.87 \pm 0.02$ & $3.87 \pm 0.02$ & $1.91 \pm 0.01$ & \\
\hline 8 & J041413.58+281249.0 & FM Tau & $8.07 \pm 0.02$ & $7.48 \pm 0.02$ & $5.21 \pm 0.02$ & $3.03 \pm 0.03$ & \\
\hline 9 & J041414.59+282757.9 & FN Tau & $7.48 \pm 0.03$ & $6.78 \pm 0.02$ & $4.09 \pm 0.02$ & $1.96 \pm 0.02$ & \\
\hline 10 & J041417.61+280609.5 & CIDA-1 & $9.05 \pm 0.02$ & $8.17 \pm 0.02$ & $5.49 \pm 0.02$ & $3.56 \pm 0.03$ & \\
\hline 11 & J041426.31+280601.9 & MHO-1 & $6.16 \pm 0.04$ & $4.81 \pm 0.03$ & $2.79 \pm 0.02$ & $0.44 \pm 0.01$ & \\
\hline 12 & J041430.55+280514.4 & MHO-3 & $6.95 \pm 0.03$ & $5.70 \pm 0.03$ & $2.58 \pm 0.02$ & $0.24 \pm 0.02$ & \\
\hline 13 & J041447.30+264626.3 & FP Tau & $8.39 \pm 0.02$ & $7.95 \pm 0.02$ & $6.47 \pm 0.02$ & $4.43 \pm 0.03$ & \\
\hline 14 & J041447.86+264810.9 & CX Tau & $8.51 \pm 0.02$ & $8.04 \pm 0.02$ & $5.65 \pm 0.02$ & $3.45 \pm 0.02$ & \\
\hline 15 & $\mathrm{~J} 041449.28+281230.4$ & FO Tau AB & $7.42 \pm 0.03$ & $6.91 \pm 0.02$ & $5.11 \pm 0.02$ & $3.05 \pm 0.02$ & \\
\hline 16 & J041535.66+284741.3 & None & $13.61 \pm 0.03$ & $12.00 \pm 0.03$ & $9.29 \pm 0.04$ & $5.84 \pm 0.04$ & Still a candidate $\mathrm{YSO}^{\mathrm{b}}$ \\
\hline 17 & J041539.16+281858.3 & $041539.1+281858$ & $8.77 \pm 0.02$ & $8.33 \pm 0.02$ & $6.86 \pm 0.02$ & $4.44 \pm 0.03$ & \\
\hline 18 & J041542.77+290959.1 & $041542.7+290959$ & $9.04 \pm 0.02$ & $8.91 \pm 0.02$ & $8.57 \pm 0.03$ & $4.76 \pm 0.02$ & \\
\hline 19 & J041558.00+274617.2 & $041557.9+274617$ & $9.85 \pm 0.02$ & $9.33 \pm 0.02$ & $7.47 \pm 0.02$ & $5.86 \pm 0.04$ & \\
\hline 20 & J041604.83+261800.9 & None & $12.36 \pm 0.03$ & $11.28 \pm 0.02$ & $8.68 \pm 0.03$ & $6.61 \pm 0.07$ & Still a candidate $\mathrm{YSO}^{\mathrm{b}}$ \\
\hline 21 & J041612.10+275638.3 & J04161210+2756385 & $9.47 \pm 0.03$ & $8.86 \pm 0.02$ & $7.68 \pm 0.02$ & $5.56 \pm 0.04$ & \\
\hline 22 & J041639.12+285848.8 & J04163911+2858491 & $10.58 \pm 0.02$ & $10.07 \pm 0.02$ & $8.86 \pm 0.03$ & $7.29 \pm 0.12$ & (SDSS spectrum: STAR -M8) \\
\hline 23 & J041749.55+281331.5 & KPNO-10 & $11.38 \pm 0.02$ & $10.55 \pm 0.02$ & $8.01 \pm 0.02$ & $6.04 \pm 0.05$ & (SDSS spectrum: STAR -M6) \\
\hline 24 & J041749.65+282936.0 & V410 X-ray 1 & $8.43 \pm 0.02$ & $7.76 \pm 0.02$ & $5.49 \pm 0.02$ & $3.83 \pm 0.03$ & \\
\hline 25 & J041803.37+244009.6 & $041803.3+244009$ & $10.01 \pm 0.03$ & $10.01 \pm 0.02$ & $9.39 \pm 0.04$ & $7.33 \pm 0.11$ & Still a candidate $\mathrm{YSO}^{\mathrm{b}}$ \\
\hline 26 & J041810.60+284447.0 & None & $12.66 \pm 0.03$ & $11.59 \pm 0.02$ & $9.11 \pm 0.04$ & $6.58 \pm 0.07$ & Still a candidate $\mathrm{YSO}^{\mathrm{b}}$ \\
\hline 27 & J041810.78+251957.2 & [GBM90]L15061 & $8.32 \pm 0.02$ & $7.93 \pm 0.02$ & $5.54 \pm 0.02$ & $3.81 \pm 0.02$ & \\
\hline 28 & J041817.10+282841.6 & V410 Anon 13 & $10.52 \pm 0.03$ & $9.84 \pm 0.02$ & $7.97 \pm 0.02$ & $6.15 \pm 0.05$ & \\
\hline 29 & J041823.20+251928.0 & 2MASX04182321+2519281 & $11.92 \pm 0.03$ & $10.90 \pm 0.02$ & $8.43 \pm 0.03$ & $6.28 \pm 0.05$ & Still a candidate $\mathrm{YSO}^{\mathrm{b}}$ \\
\hline 30 & J041831.12+281628.8 & DD Tau AB & $6.83 \pm 0.03$ & $5.78 \pm 0.02$ & $3.72 \pm 0.02$ & $1.80 \pm 0.02$ & \\
\hline 31 & J041831.60+281658.3 & CZ Tau AB & $8.65 \pm 0.02$ & $7.49 \pm 0.02$ & $3.85 \pm 0.02$ & $2.13 \pm 0.02$ & \\
\hline 32 & $\mathrm{~J} 041832.03+283115.1$ & IRAS $04154+2823$ & $7.78 \pm 0.02$ & $6.52 \pm 0.02$ & $4.28 \pm 0.02$ & $1.99 \pm 0.02$ & \\
\hline 33 & $\mathrm{~J} 041834.44+283030.0$ & V410 X-ray 2 & $8.65 \pm 0.02$ & $8.01 \pm 0.02$ & $6.72 \pm 0.02$ & $3.58 \pm 0.02$ & \\
\hline 34 & J041841.32+282724.7 & LR1 & $9.98 \pm 0.02$ & $8.93 \pm 0.02$ & $7.48 \pm 0.03$ & $4.82 \pm 0.03$ & \\
\hline 35 & $\mathrm{~J} 041851.47+282026.2$ & CoKu Tau/1 & $10.27 \pm 0.02$ & $8.47 \pm 0.02$ & $3.84 \pm 0.02$ & $1.01 \pm 0.02$ & (SDSS spectrum: GALAXY-STARBURST) \\
\hline 36 & $\mathrm{~J} 041858.07+235030.8$ & None & $12.95 \pm 0.03$ & $11.78 \pm 0.02$ & $9.28 \pm 0.04$ & $7.41 \pm 0.12$ & Still a candidate $\mathrm{YSO}^{\mathrm{b}}$ \\
\hline 37 & $\mathrm{~J} 041858.13+281223.1$ & IRAS $04158+2805$ & $9.47 \pm 0.03$ & $8.32 \pm 0.02$ & $5.70 \pm 0.02$ & $2.99 \pm 0.02$ & \\
\hline 38 & $\mathrm{~J} 041858.99+255740.0$ & None & $13.37 \pm 0.03$ & $11.98 \pm 0.02$ & $8.64 \pm 0.03$ & $6.06 \pm 0.04$ & Still a candidate $\mathrm{YSO}^{\mathrm{b}}$ \\
\hline 39 & J041901.10+281941.8 & V410 X-ray 6 & $8.98 \pm 0.03$ & $8.64 \pm 0.02$ & $7.20 \pm 0.02$ & $3.89 \pm 0.03$ & \\
\hline 40 & J041915.83+290626.6 & BP Tau & $7.08 \pm 0.03$ & $6.64 \pm 0.02$ & $4.78 \pm 0.02$ & $2.74 \pm 0.02$ & \\
\hline 41 & J041935.46+282721.4 & FR Tau & $9.55 \pm 0.03$ & $8.77 \pm 0.02$ & $6.33 \pm 0.02$ & $4.86 \pm 0.03$ & \\
\hline 42 & J041940.48+270100.7 & None & $13.16 \pm 0.03$ & $11.94 \pm 0.03$ & $9.21 \pm 0.05$ & $6.88 \pm 0.10$ & Still a candidate $\mathrm{YSO}^{\mathrm{b}}$ \\
\hline 43 & J041941.49+271606.8 & HH390 star & $11.65 \pm 0.03$ & $10.36 \pm 0.02$ & $7.43 \pm 0.02$ & $3.25 \pm 0.02$ & \\
\hline 44 & J041946.58+271255.0 & J04194657+2712552 & $10.81 \pm 0.03$ & $9.36 \pm 0.02$ & $7.05 \pm 0.02$ & $4.14 \pm 0.03$ & \\
\hline 45 & J041958.45+270957.0 & IRAS 04169+2702 & $8.90 \pm 0.02$ & $6.96 \pm 0.02$ & $3.93 \pm 0.02$ & $0.81 \pm 0.02$ & \\
\hline
\end{tabular}


Table 1

(Continued)

\begin{tabular}{|c|c|c|c|c|c|c|c|}
\hline $\begin{array}{l}\text { Line } \\
\text { Num. }\end{array}$ & Catalog Number & Name & $\begin{array}{l}\text { W1 }(3.4 \mu \mathrm{m}) \\
(\mathrm{mag})\end{array}$ & $\begin{array}{l}\text { W2 }(4.6 \mu \mathrm{m}) \\
(\mathrm{mag})\end{array}$ & $\begin{array}{l}\text { W3 }(12 \mu \mathrm{m}) \\
\quad(\mathrm{mag})\end{array}$ & $\begin{array}{l}\text { W4 }(22 \mu \mathrm{m}) \\
\quad(\mathrm{mag})\end{array}$ & Notes \\
\hline 46 & J042025.55+270035.4 & J04202555+2700355 & $11.19 \pm 0.03$ & $10.71 \pm 0.02$ & $8.64 \pm 0.03$ & $6.14 \pm 0.06$ & (SDSS spectrum: STAR -M7) \\
\hline 47 & $\mathrm{~J} 042025.83+281923.5$ & $\mathrm{~J} 04202583+2819237$ & $9.45 \pm 0.02$ & $8.38 \pm 0.02$ & $6.01 \pm 0.02$ & $4.05 \pm 0.03$ & \\
\hline 48 & $\mathrm{~J} 042026.06+280408.8$ & J04202606+2804089 & $9.46 \pm 0.03$ & $9.07 \pm 0.02$ & $6.53 \pm 0.02$ & $3.73 \pm 0.03$ & \\
\hline 49 & $\mathrm{~J} 042107.96+270220.3$ & CFHT-19 & $8.02 \pm 0.02$ & $6.87 \pm 0.02$ & $4.22 \pm 0.02$ & $1.84 \pm 0.02$ & \\
\hline 50 & J042110.39+270137.1 & IRAS 04181+2654B & $9.57 \pm 0.02$ & $8.12 \pm 0.02$ & $5.66 \pm 0.02$ & $2.87 \pm 0.02$ & \\
\hline 51 & $\mathrm{~J} 042111.46+270109.3$ & IRAS 04181+2654A & $9.03 \pm 0.03$ & $7.57 \pm 0.02$ & $4.84 \pm 0.02$ & $1.78 \pm 0.02$ & \\
\hline 52 & J042143.23+193413.2 & $04187+1927$ & $7.08 \pm 0.03$ & $6.14 \pm 0.02$ & $3.66 \pm 0.02$ & $1.94 \pm 0.02$ & \\
\hline 53 & J042155.63+275505.9 & DE Tau & $7.07 \pm 0.03$ & $6.53 \pm 0.02$ & $4.86 \pm 0.02$ & $2.80 \pm 0.03$ & \\
\hline 54 & J042159.44+193205.9 & TTau & $3.86 \pm 0.06$ & $2.20 \pm 0.01$ & $0.10 \pm 0.00$ & $-2.13 \pm 0.02$ & \\
\hline 55 & $\mathrm{~J} 042200.42+153021.2$ & 2MASX04220037+1530212 & $10.51 \pm 0.03$ & $8.35 \pm 0.02$ & $5.34 \pm 0.01$ & $2.35 \pm 0.03$ & \\
\hline 56 & $\mathrm{~J} 042247.87+264552.7$ & IRAS $04196+2638$ & $8.14 \pm 0.02$ & $7.36 \pm 0.02$ & $5.49 \pm 0.07$ & $3.27 \pm 0.03$ & \\
\hline 57 & $\mathrm{~J} 042307.77+280557.1$ & IRAS $04200+2759$ & $8.80 \pm 0.02$ & $7.98 \pm 0.02$ & $5.52 \pm 0.02$ & $3.38 \pm 0.03$ & (SDSS spectrum: STAR -M2) \\
\hline 58 & $\mathrm{~J} 042335.39+250302.5$ & FUTau & $8.62 \pm 0.02$ & $7.85 \pm 0.02$ & $6.05 \pm 0.02$ & $4.65 \pm 0.03$ & (SDSS spectrum: STAR -M8) \\
\hline 59 & J042444.58+261014.0 & IRAS $04216+2603$ & $8.09 \pm 0.02$ & $7.36 \pm 0.02$ & $5.41 \pm 0.02$ & $3.49 \pm 0.02$ & \\
\hline 60 & J042457.08+271156.2 & IP Tau & $7.75 \pm 0.02$ & $7.26 \pm 0.02$ & $5.38 \pm 0.02$ & $3.59 \pm 0.03$ & \\
\hline 61 & J042519.16+234716.7 & HD27923 & $7.54 \pm 0.03$ & $7.57 \pm 0.02$ & $7.58 \pm 0.02$ & $1.33 \pm 0.01$ & Still a candidate $\mathrm{YSO}^{\mathrm{b}}$ \\
\hline 62 & $\mathrm{~J} 042629.39+262413.4$ & KPNO-3 & $11.56 \pm 0.03$ & $10.94 \pm 0.02$ & $8.76 \pm 0.03$ & $6.91 \pm 0.09$ & (SDSS spectrum: STAR -M8) \\
\hline 63 & $\mathrm{~J} 042653.50+260654.1$ & FV Tau AB & $6.78 \pm 0.03$ & $5.58 \pm 0.02$ & $3.21 \pm 0.01$ & $1.37 \pm 0.01$ & \\
\hline 64 & $\mathrm{~J} 042656.25+244335.1$ & IRAS $04239+2436$ & $8.13 \pm 0.02$ & $6.05 \pm 0.02$ & $3.12 \pm 0.02$ & $0.17 \pm 0.03$ & \\
\hline 65 & $\mathrm{~J} 042657.31+260628.1$ & KPNO-13 & $8.94 \pm 0.03$ & $8.27 \pm 0.02$ & $6.50 \pm 0.03$ & $4.95 \pm 0.08$ & (SDSS spectrum: STAR -M7) \\
\hline 66 & $\mathrm{~J} 042702.56+260530.4$ & DGTauB & $8.97 \pm 0.03$ & $6.71 \pm 0.02$ & $3.41 \pm 0.02$ & $0.78 \pm 0.02$ & \\
\hline 67 & $\mathrm{~J} 042702.79+254222.2$ & DF Tau AB & $5.89 \pm 0.05$ & $5.03 \pm 0.03$ & $3.80 \pm 0.02$ & $2.31 \pm 0.02$ & \\
\hline 68 & $\mathrm{~J} 042704.69+260615.9$ & DG Tau A & $6.18 \pm 0.04$ & $4.98 \pm 0.03$ & $2.26 \pm 0.01$ & $-0.19 \pm 0.02$ & \\
\hline 69 & $\mathrm{~J} 042730.27+244123.6$ & 2MASX04273023+2441232 & $11.75 \pm 0.03$ & $11.49 \pm 0.03$ & $8.35 \pm 0.03$ & $6.31 \pm 0.10$ & Still a candidate $\mathrm{YSO}^{\mathrm{b}}$ \\
\hline 70 & $\mathrm{~J} 042757.31+261918.1$ & IRAS $04248+2612 \mathrm{AB}$ & $9.81 \pm 0.02$ & $8.75 \pm 0.02$ & $5.45 \pm 0.02$ & $2.35 \pm 0.01$ & \\
\hline 71 & $\mathrm{~J} 042810.38+243553.7$ & None & $13.99 \pm 0.03$ & $12.44 \pm 0.03$ & $9.79 \pm 0.05$ & $7.61 \pm 0.16$ & Still a candidate $\mathrm{YSO}^{\mathrm{b}}$ \\
\hline 72 & $\mathrm{~J} 042842.62+271403.5$ & $\mathrm{~J} 04284263+2714039 \mathrm{AB}$ & $9.79 \pm 0.02$ & $9.37 \pm 0.02$ & $8.09 \pm 0.03$ & $6.18 \pm 0.05$ & \\
\hline 73 & J042904.98+264907.1 & IRAS $04260+2642$ & $10.57 \pm 0.02$ & $9.53 \pm 0.02$ & $7.00 \pm 0.02$ & $3.65 \pm 0.02$ & \\
\hline 74 & $\mathrm{~J} 042920.82+274207.3$ & IRAS $04262+2735$ & $6.55 \pm 0.04$ & $6.34 \pm 0.02$ & $3.84 \pm 0.02$ & $3.02 \pm 0.02$ & \\
\hline 75 & J042921.65+270125.7 & IRAS $04263+2654$ & $8.27 \pm 0.03$ & $7.62 \pm 0.02$ & $5.77 \pm 0.02$ & $3.50 \pm 0.02$ & \\
\hline 76 & J042923.73+243300.9 & GV Tau AB & $5.95 \pm 0.04$ & $3.24 \pm 0.04$ & $0.30 \pm 0.00$ & $-1.63 \pm 0.01$ & (SDSS spectrum: GALAXY-STARBURST) \\
\hline 77 & J042930.08+243954.9 & IRAS 04264+2433 & $10.28 \pm 0.02$ & $9.05 \pm 0.02$ & $4.35 \pm 0.02$ & $1.13 \pm 0.01$ & \\
\hline 78 & J042932.08+243059.4 & None & $11.06 \pm 0.03$ & $9.20 \pm 0.02$ & $6.29 \pm 0.02$ & $4.04 \pm 0.04$ & \\
\hline 79 & J042936.06+243555.4 & J04293606+2435556 & $8.12 \pm 0.02$ & $7.64 \pm 0.02$ & $6.39 \pm 0.02$ & $4.44 \pm 0.03$ & \\
\hline 80 & $\mathrm{~J} 042941.55+263258.0$ & DH Tau AB & $7.42 \pm 0.03$ & $6.99 \pm 0.02$ & $5.73 \pm 0.02$ & $3.07 \pm 0.02$ & \\
\hline 81 & $\mathrm{~J} 042951.55+260644.7$ & IQ Tau & $7.21 \pm 0.03$ & $6.67 \pm 0.02$ & $4.87 \pm 0.02$ & $3.01 \pm 0.02$ & \\
\hline 82 & $\mathrm{~J} 042954.22+175403.8$ & $04295422+1754041$ & $10.12 \pm 0.02$ & $9.47 \pm 0.02$ & $8.13 \pm 0.03$ & $6.42 \pm 0.06$ & \\
\hline 83 & $\mathrm{~J} 042959.50+243307.5$ & CFHT-20 & $9.09 \pm 0.02$ & $8.45 \pm 0.02$ & $6.84 \pm 0.02$ & $4.88 \pm 0.03$ & (SDSS spectrum: STAR -M8) \\
\hline 84 & J043003.99+181349.4 & UXTau & $6.99 \pm 0.03$ & $6.61 \pm 0.02$ & $5.69 \pm 0.01$ & $2.17 \pm 0.02$ & \\
\hline 85 & J043029.62+242644.9 & FX Tau AB & $7.49 \pm 0.03$ & $7.09 \pm 0.02$ & $5.09 \pm 0.02$ & $3.23 \pm 0.02$ & \\
\hline 86 & $\mathrm{~J} 043044.25+260124.4$ & DK Tau AB & $6.12 \pm 0.04$ & $5.38 \pm 0.03$ & $3.59 \pm 0.01$ & $1.84 \pm 0.01$ & \\
\hline 87 & J043051.38+244222.2 & ZZ Tau AB & $8.09 \pm 0.02$ & $7.66 \pm 0.02$ & $5.94 \pm 0.02$ & $4.33 \pm 0.03$ & \\
\hline 88 & J043051.71+244147.4 & ZZ Tau IRS & $9.01 \pm 0.02$ & $7.16 \pm 0.02$ & $4.43 \pm 0.02$ & $1.95 \pm 0.02$ & \\
\hline 89 & J043134.12+180804.9 & 2MASX04313407+1808048 & $7.75 \pm 0.02$ & $5.55 \pm 0.02$ & $0.86 \pm 0.01$ & $-2.37 \pm 0.01$ & \\
\hline 90 & J043136.14+181343.1 & LkHa358 & $8.17 \pm 0.02$ & $7.16 \pm 0.02$ & $4.96 \pm 0.02$ & $2.39 \pm 0.03$ & \\
\hline 91 & J043138.42+181357.6 & HLTau & $5.42 \pm 0.05$ & $3.34 \pm 0.03$ & $1.52 \pm 0.01$ & $-1.23 \pm 0.01$ & \\
\hline 92 & $\mathrm{~J} 043140.09+181357.0$ & XZTau & $5.90 \pm 0.05$ & $4.64 \pm 0.04$ & $2.65 \pm 0.02$ & $0.53 \pm 0.02$ & \\
\hline
\end{tabular}


Table 1

(Continued)

\begin{tabular}{|c|c|c|c|c|c|c|c|}
\hline $\begin{array}{l}\text { Line } \\
\text { Num. }\end{array}$ & Catalog Number & Name & $\begin{array}{l}\text { W1 }(3.4 \mu \mathrm{m}) \\
\quad(\mathrm{mag})\end{array}$ & $\begin{array}{l}\text { W2 }(4.6 \mu \mathrm{m}) \\
\quad(\mathrm{mag})\end{array}$ & $\begin{array}{l}\text { W3 }(12 \mu \mathrm{m}) \\
\quad(\mathrm{mag})\end{array}$ & $\begin{array}{c}\text { W4 }(22 \mu \mathrm{m}) \\
\quad(\mathrm{mag})\end{array}$ & Notes \\
\hline 93 & J043144.47+180831.4 & L1551NE & $9.31 \pm 0.02$ & $6.88 \pm 0.02$ & $4.18 \pm 0.02$ & $0.15 \pm 0.02$ & \\
\hline 94 & $\mathrm{~J} 043150.56+242417.6$ & HK Tau AB & $7.81 \pm 0.03$ & $7.35 \pm 0.02$ & $5.49 \pm 0.02$ & $2.46 \pm 0.02$ & \\
\hline 95 & J043157.79+182136.9 & V710Tau & $7.55 \pm 0.02$ & $7.14 \pm 0.02$ & $5.54 \pm 0.02$ & $3.76 \pm 0.03$ & \\
\hline 96 & J043159.68+182130.3 & J04315968+1821305 & $8.84 \pm 0.02$ & $7.98 \pm 0.02$ & $6.55 \pm 0.02$ & $4.73 \pm 0.03$ & \\
\hline 97 & $\mathrm{~J} 043215.41+242859.5$ & Haro6-13 & $6.63 \pm 0.03$ & $5.80 \pm 0.03$ & $3.60 \pm 0.02$ & $0.89 \pm 0.01$ & \\
\hline 98 & J043216.06+181246.3 & MHO5 & $9.61 \pm 0.03$ & $9.03 \pm 0.02$ & $7.01 \pm 0.02$ & $5.70 \pm 0.05$ & \\
\hline 99 & J043222.11+182742.6 & MHO6 & $10.22 \pm 0.02$ & $9.76 \pm 0.02$ & $8.45 \pm 0.03$ & $6.63 \pm 0.08$ & \\
\hline 100 & $\mathrm{~J} 043224.15+225108.2$ & $043224.1+225108$ & $10.17 \pm 0.02$ & $9.62 \pm 0.02$ & $7.60 \pm 0.02$ & $6.20 \pm 0.05$ & \\
\hline 101 & J043230.35+173140.6 & GGTauAa & $6.67 \pm 0.03$ & $6.13 \pm 0.02$ & $4.08 \pm 0.01$ & $2.13 \pm 0.01$ & \\
\hline 102 & J043230.57+241957.3 & FY Tau & $7.31 \pm 0.03$ & $6.78 \pm 0.03$ & $5.58 \pm 0.02$ & $4.03 \pm 0.02$ & \\
\hline 103 & $\mathrm{~J} 043232.06+225726.3$ & IRAS 04295+2251 & $8.61 \pm 0.02$ & $6.84 \pm 0.02$ & $3.90 \pm 0.02$ & $1.53 \pm 0.02$ & \\
\hline 104 & J043241.08+180923.8 & 2MASSJ04324107+1809239 & $9.86 \pm 0.03$ & $9.36 \pm 0.02$ & $8.00 \pm 0.02$ & $6.55 \pm 0.08$ & Known YSO but not traditional Taurus member \\
\hline 105 & $\mathrm{~J} 043243.00+255230.8$ & UZ Tau Aab & $6.36 \pm 0.04$ & $5.72 \pm 0.02$ & $3.66 \pm 0.02$ & $1.85 \pm 0.02$ & \\
\hline 106 & J043249.11+225302.7 & JH112 & $7.41 \pm 0.03$ & $6.88 \pm 0.02$ & $4.69 \pm 0.02$ & $2.68 \pm 0.02$ & \\
\hline 107 & J043304.22+292149.9 & HD282276 & $7.87 \pm 0.02$ & $7.71 \pm 0.02$ & $5.76 \pm 0.02$ & $2.62 \pm 0.02$ & Still a candidate $\mathrm{YSO}^{\mathrm{b}}$ \\
\hline 108 & J043306.21+240933.7 & GH Tau AB & $7.34 \pm 0.03$ & $6.87 \pm 0.02$ & $5.28 \pm 0.02$ & $3.28 \pm 0.02$ & \\
\hline 109 & J043306.62+240954.9 & V807 Tau AB & $6.51 \pm 0.04$ & $6.14 \pm 0.02$ & $5.16 \pm 0.02$ & $2.97 \pm 0.02$ & \\
\hline 110 & $\mathrm{~J} 043314.36+261423.2$ & IRAS 04301+2608 & $11.98 \pm 0.02$ & $11.13 \pm 0.02$ & $7.14 \pm 0.02$ & $3.55 \pm 0.02$ & \\
\hline 111 & $\mathrm{~J} 043316.47+225320.4$ & IRAS $04302+2247$ & $10.24 \pm 0.02$ & $9.34 \pm 0.02$ & $8.83 \pm 0.03$ & $3.99 \pm 0.02$ & \\
\hline 112 & $\mathrm{~J} 043316.60+262724.3$ & None & $13.39 \pm 0.03$ & $12.33 \pm 0.03$ & $9.64 \pm 0.05$ & $7.12 \pm 0.10$ & Still a candidate $\mathrm{YSO}^{\mathrm{b}}$ \\
\hline 113 & J043332.80+180043.4 & None & $9.38 \pm 0.03$ & $8.93 \pm 0.02$ & $6.46 \pm 0.02$ & $4.72 \pm 0.03$ & \\
\hline 114 & $\mathrm{~J} 043334.06+242117.0$ & GI Tau & $7.10 \pm 0.03$ & $6.37 \pm 0.02$ & $4.01 \pm 0.02$ & $2.54 \pm 0.03$ & \\
\hline 115 & J043334.55+242105.9 & GK Tau & $6.42 \pm 0.04$ & $5.80 \pm 0.03$ & $3.70 \pm 0.02$ & $1.55 \pm 0.02$ & \\
\hline 116 & J043336.80+260949.0 & IS Tau AB & $8.06 \pm 0.02$ & $7.41 \pm 0.02$ & $5.24 \pm 0.02$ & $3.74 \pm 0.02$ & \\
\hline 117 & J043339.06+222720.6 & $043339.0+222720$ & $10.16 \pm 0.02$ & $9.73 \pm 0.02$ & $8.06 \pm 0.03$ & $5.04 \pm 0.03$ & \\
\hline 118 & $\mathrm{~J} 043339.07+252038.0$ & DL Tau & $6.98 \pm 0.03$ & $6.24 \pm 0.02$ & $4.31 \pm 0.02$ & $2.44 \pm 0.02$ & \\
\hline 119 & J043339.36+175152.1 & HNTau & $7.26 \pm 0.03$ & $6.41 \pm 0.02$ & $4.15 \pm 0.02$ & $2.18 \pm 0.02$ & \\
\hline 120 & J043341.73+175039.9 & J04334171+1750402 & $10.07 \pm 0.03$ & $9.73 \pm 0.02$ & $8.37 \pm 0.03$ & $6.42 \pm 0.08$ & \\
\hline 121 & $\mathrm{~J} 043344.66+261500.3$ & J04334465+2615005 & $9.19 \pm 0.03$ & $8.41 \pm 0.02$ & $6.60 \pm 0.02$ & $4.78 \pm 0.03$ & \\
\hline 122 & J043348.73+181009.8 & DMTau & $9.47 \pm 0.03$ & $9.30 \pm 0.02$ & $7.04 \pm 0.02$ & $3.60 \pm 0.02$ & \\
\hline 123 & $\mathrm{~J} 043352.01+225030.0$ & CI Tau & $6.78 \pm 0.03$ & $6.07 \pm 0.02$ & $4.34 \pm 0.02$ & $2.47 \pm 0.02$ & \\
\hline 124 & $\mathrm{~J} 043354.69+261327.0$ & IT Tau AB & $7.45 \pm 0.03$ & $6.89 \pm 0.02$ & $5.22 \pm 0.02$ & $3.61 \pm 0.02$ & \\
\hline 125 & J043455.42+242852.9 & AA Tau & $7.43 \pm 0.03$ & $6.76 \pm 0.02$ & $4.62 \pm 0.02$ & $2.54 \pm 0.02$ & \\
\hline 126 & J043520.21+223214.4 & HO Tau & $8.97 \pm 0.02$ & $8.50 \pm 0.02$ & $6.88 \pm 0.02$ & $5.01 \pm 0.03$ & \\
\hline 127 & J043527.37+241458.7 & DN Tau & $7.78 \pm 0.02$ & $7.29 \pm 0.02$ & $5.12 \pm 0.02$ & $3.08 \pm 0.02$ & \\
\hline 128 & J043535.40+240819.9 & IRAS 04325+2402 A & $9.84 \pm 0.02$ & $8.72 \pm 0.02$ & $6.36 \pm 0.02$ & $1.64 \pm 0.01$ & \\
\hline 129 & J043540.95+241108.4 & CoKu Tau/3 AB & $7.54 \pm 0.03$ & $6.71 \pm 0.02$ & $4.78 \pm 0.02$ & $3.33 \pm 0.02$ & \\
\hline 130 & J043547.34+225021.6 & HQTau & $6.82 \pm 0.03$ & $6.11 \pm 0.02$ & $3.45 \pm 0.02$ & $1.54 \pm 0.01$ & \\
\hline 131 & J043552.78+225423.1 & HP Tau AB & $6.54 \pm 0.04$ & $5.86 \pm 0.03$ & $3.62 \pm 0.02$ & $1.47 \pm 0.02$ & \\
\hline 132 & J043556.83+225435.8 & Haro 6-28 AB & $8.83 \pm 0.03$ & $8.18 \pm 0.02$ & $6.31 \pm 0.02$ & $4.41 \pm 0.03$ & \\
\hline 133 & J043601.32+172611.9 & 2MASSJ04360131+1726120 & $8.62 \pm 0.02$ & $8.06 \pm 0.02$ & $7.16 \pm 0.02$ & $5.40 \pm 0.04$ & Known YSO but not traditional Taurus member \\
\hline 134 & $\mathrm{~J} 043630.81+184215.3$ & HD285893 & $7.16 \pm 0.03$ & $6.55 \pm 0.02$ & $3.73 \pm 0.02$ & $1.46 \pm 0.01$ & Known YSO but not traditional Taurus member \\
\hline 135 & J043642.05+265339.8 & None & $13.22 \pm 0.03$ & $11.49 \pm 0.02$ & $9.07 \pm 0.03$ & $6.47 \pm 0.07$ & Still a candidate $\mathrm{YSO}^{\mathrm{b}}$ \\
\hline 136 & $\mathrm{~J} 043756.70+254623.0$ & ITG 1 & $12.11 \pm 0.03$ & $11.29 \pm 0.02$ & $8.89 \pm 0.03$ & $7.07 \pm 0.10$ & \\
\hline 137 & J043814.87+261139.8 & J04381486+2611399 & $11.10 \pm 0.02$ & $10.06 \pm 0.02$ & $7.87 \pm 0.02$ & $5.06 \pm 0.03$ & \\
\hline 138 & $\mathrm{~J} 043821.33+260913.6$ & GM Tau & $9.52 \pm 0.02$ & $8.83 \pm 0.02$ & $7.07 \pm 0.02$ & $5.34 \pm 0.04$ & \\
\hline
\end{tabular}


Table 1

(Continued)

\begin{tabular}{|c|c|c|c|c|c|c|c|}
\hline $\begin{array}{l}\text { Line } \\
\text { Num. }\end{array}$ & Catalog Number & Name & $\begin{array}{c}\text { W1 }(3.4 \mu \mathrm{m}) \\
(\mathrm{mag})\end{array}$ & $\begin{array}{c}\text { W2 }(4.6 \mu \mathrm{m}) \\
(\mathrm{mag})\end{array}$ & $\begin{array}{l}\text { W3 }(12 \mu \mathrm{m}) \\
\quad(\mathrm{mag})\end{array}$ & $\begin{array}{c}\text { W4 }(22 \mu \mathrm{m}) \\
(\mathrm{mag})\end{array}$ & Notes \\
\hline 139 & J043828.58+261049.4 & DO Tau & $6.31 \pm 0.04$ & $5.42 \pm 0.03$ & $3.43 \pm 0.02$ & $1.20 \pm 0.01$ & \\
\hline 140 & $\mathrm{~J} 043835.31+261038.8$ & HV Tau AB & $7.77 \pm 0.02$ & $7.51 \pm 0.02$ & $6.71 \pm 0.02$ & $3.62 \pm 0.03$ & \\
\hline 141 & $\mathrm{~J} 043858.60+233635.0$ & $\mathrm{~J} 0438586+2336352$ & $10.72 \pm 0.03$ & $10.19 \pm 0.02$ & $8.32 \pm 0.03$ & $6.55 \pm 0.07$ & \\
\hline 142 & $\mathrm{~J} 043901.63+233602.7$ & $\mathrm{~J} 0439016+2336030$ & $9.87 \pm 0.02$ & $9.46 \pm 0.02$ & $7.95 \pm 0.02$ & $6.39 \pm 0.06$ & \\
\hline 143 & $\mathrm{~J} 043903.95+254426.2$ & CFHT- 6 & $10.98 \pm 0.02$ & $10.38 \pm 0.02$ & $8.33 \pm 0.03$ & $6.56 \pm 0.07$ & (SDSS spectrum: STAR -M9) \\
\hline 144 & J043905.24+233744.9 & $\mathrm{J} 04390525+2337450$ & $10.63 \pm 0.02$ & $10.02 \pm 0.02$ & $7.28 \pm 0.02$ & $3.69 \pm 0.03$ & \\
\hline 145 & J043913.89+255320.9 & IRAS $04361+2547 \mathrm{AB}$ & $8.68 \pm 0.02$ & $7.08 \pm 0.02$ & $2.76 \pm 0.02$ & $-1.01 \pm 0.01$ & \\
\hline 146 & $\mathrm{~J} 043917.42+224753.3$ & VYTau & $8.49 \pm 0.02$ & $8.03 \pm 0.02$ & $6.19 \pm 0.02$ & $4.76 \pm 0.03$ & \\
\hline 147 & $\mathrm{~J} 043917.79+222103.3$ & $\mathrm{LkCa} 15$ & $7.49 \pm 0.03$ & $7.15 \pm 0.02$ & $5.67 \pm 0.02$ & $3.60 \pm 0.03$ & \\
\hline 148 & J043920.91+254501.9 & GN Tau B & $7.13 \pm 0.03$ & $6.51 \pm 0.02$ & $4.88 \pm 0.02$ & $3.08 \pm 0.02$ & \\
\hline 149 & J043933.64+235921.0 & J04393364+2359212 & $9.78 \pm 0.02$ & $9.15 \pm 0.02$ & $6.97 \pm 0.02$ & $5.26 \pm 0.03$ & \\
\hline 150 & J043935.18+254144.4 & IRAS $04365+2535$ & $7.96 \pm 0.02$ & $5.61 \pm 0.03$ & $3.42 \pm 0.02$ & $0.17 \pm 0.01$ & \\
\hline 151 & $\mathrm{~J} 043944.87+260152.6$ & ITG 15 & $8.41 \pm 0.02$ & $7.78 \pm 0.02$ & $6.26 \pm 0.02$ & $4.09 \pm 0.03$ & \\
\hline 152 & $\mathrm{~J} 043947.47+260140.6$ & CFHT-4 & $9.83 \pm 0.03$ & $8.97 \pm 0.02$ & $6.99 \pm 0.02$ & $5.17 \pm 0.04$ & (SDSS spectrum: STAR -M9) \\
\hline 153 & J043953.96+260309.7 & IRAS 04368+2557 & $13.03 \pm 0.05$ & $9.75 \pm 0.03$ & $8.74 \pm 0.04$ & $3.12 \pm 0.02$ & \\
\hline 154 & $\mathrm{~J} 044000.67+235820.9$ & $\mathrm{~J} 04400067+2358211$ & $11.15 \pm 0.04$ & $10.66 \pm 0.03$ & $8.91 \pm 0.03$ & $6.63 \pm 0.07$ & \\
\hline 155 & $\mathrm{~J} 044007.99+260525.3$ & IRAS $04370+2559$ & $8.21 \pm 0.02$ & $7.27 \pm 0.02$ & $4.85 \pm 0.02$ & $2.74 \pm 0.02$ & \\
\hline 156 & $\mathrm{~J} 044049.50+255119.1$ & JH223 & $8.90 \pm 0.02$ & $8.43 \pm 0.02$ & $7.07 \pm 0.02$ & $5.30 \pm 0.04$ & \\
\hline 157 & $\mathrm{~J} 044108.26+255607.3$ & ITG $33 \mathrm{~A}$ & $9.76 \pm 0.02$ & $8.87 \pm 0.02$ & $6.63 \pm 0.02$ & $4.50 \pm 0.03$ & \\
\hline 158 & $\mathrm{~J} 044110.78+255511.5$ & ITG 34 & $11.03 \pm 0.03$ & $10.31 \pm 0.02$ & $8.31 \pm 0.04$ & $6.71 \pm 0.10$ & \\
\hline 159 & $\mathrm{~J} 044112.68+254635.0$ & IRAS $04381+2540$ & $9.85 \pm 0.02$ & $7.67 \pm 0.02$ & $4.85 \pm 0.02$ & $1.71 \pm 0.02$ & \\
\hline 160 & $\mathrm{~J} 044116.81+283959.8$ & CoKuTau4 & $8.50 \pm 0.03$ & $8.35 \pm 0.02$ & $6.66 \pm 0.02$ & $2.47 \pm 0.02$ & \\
\hline 161 & $\mathrm{~J} 044124.65+254352.7$ & ITG 40 & $10.78 \pm 0.02$ & $9.75 \pm 0.02$ & $8.29 \pm 0.03$ & $5.63 \pm 0.04$ & \\
\hline 162 & $\mathrm{~J} 044125.75+254349.3$ & IRA04412575+2543492 & $14.48 \pm 0.04$ & $12.33 \pm 0.03$ & $9.35 \pm 0.04$ & $7.05 \pm 0.10$ & Still a candidate $\mathrm{YSO}^{\mathrm{b}}$ \\
\hline 163 & $\mathrm{~J} 044138.82+255626.7$ & IRAS $04385+2550$ & $8.16 \pm 0.02$ & $7.36 \pm 0.02$ & $4.55 \pm 0.01$ & $2.05 \pm 0.01$ & \\
\hline 164 & $\mathrm{~J} 044148.24+253430.4$ & $\mathrm{~J} 04414825+2534304$ & $11.60 \pm 0.03$ & $10.78 \pm 0.02$ & $8.53 \pm 0.03$ & $6.29 \pm 0.06$ & (SDSS spectrum: STAR -M9) \\
\hline 165 & $\mathrm{~J} 044207.77+252311.5$ & V955 Tau Ab & $6.96 \pm 0.03$ & $6.31 \pm 0.02$ & $4.49 \pm 0.02$ & $2.76 \pm 0.02$ & \\
\hline 166 & $\mathrm{~J} 044221.02+252034.2$ & CIDA-7 & $9.68 \pm 0.03$ & $9.03 \pm 0.02$ & $6.83 \pm 0.02$ & $4.38 \pm 0.02$ & \\
\hline 167 & $\mathrm{~J} 044237.68+251537.2$ & DP Tau & $7.32 \pm 0.03$ & $6.49 \pm 0.02$ & $4.23 \pm 0.02$ & $2.01 \pm 0.02$ & \\
\hline 168 & $\mathrm{~J} 044303.08+252018.6$ & GO Tau & $9.02 \pm 0.02$ & $8.57 \pm 0.02$ & $6.58 \pm 0.02$ & $4.43 \pm 0.03$ & \\
\hline 169 & $\mathrm{~J} 044315.85+235358.2$ & None & $13.15 \pm 0.03$ & $11.73 \pm 0.03$ & $9.12 \pm 0.03$ & $6.66 \pm 0.06$ & Still a candidate $\mathrm{YSO}^{\mathrm{b}}$ \\
\hline 170 & $\mathrm{~J} 044320.22+294005.8$ & CIDA-14 & $9.11 \pm 0.02$ & $8.65 \pm 0.02$ & $6.86 \pm 0.02$ & $5.64 \pm 0.04$ & \\
\hline 171 & $\mathrm{~J} 044427.14+251216.2$ & IRAS $04414+2506$ & $10.06 \pm 0.03$ & $9.36 \pm 0.02$ & $6.73 \pm 0.02$ & $4.34 \pm 0.03$ & \\
\hline 172 & $\mathrm{~J} 044653.05+170000.1$ & DQTau & $7.07 \pm 0.03$ & $6.45 \pm 0.02$ & $4.31 \pm 0.02$ & $2.28 \pm 0.02$ & \\
\hline 173 & $\mathrm{~J} 044659.01+170238.4$ & Haro6-37 & $6.35 \pm 0.04$ & $5.69 \pm 0.03$ & $4.11 \pm 0.02$ & $2.45 \pm 0.02$ & \\
\hline 174 & $\mathrm{~J} 044748.59+292511.0$ & DSTau & $7.39 \pm 0.03$ & $6.84 \pm 0.02$ & $5.18 \pm 0.02$ & $3.68 \pm 0.02$ & \\
\hline 175 & $\mathrm{~J} 044832.35+234746.9$ & None & $13.01 \pm 0.03$ & $11.72 \pm 0.02$ & $8.69 \pm 0.03$ & $6.32 \pm 0.05$ & Still a candidate $\mathrm{YSO}^{\mathrm{b}}$ \\
\hline 176 & $\mathrm{~J} 045147.36+304713.1$ & UYAur & $6.13 \pm 0.04$ & $4.92 \pm 0.03$ & $2.46 \pm 0.02$ & $0.38 \pm 0.01$ & \\
\hline 177 & $\mathrm{~J} 045206.67+304717.2$ & IRAS $04489+3042$ & $8.84 \pm 0.03$ & $7.56 \pm 0.02$ & $5.09 \pm 0.02$ & $2.52 \pm 0.02$ & \\
\hline 178 & $\mathrm{~J} 045209.70+303745.2$ & Haro6-39 & $9.95 \pm 0.02$ & $8.88 \pm 0.02$ & $6.18 \pm 0.02$ & $3.55 \pm 0.02$ & \\
\hline 179 & $\mathrm{~J} 045423.67+170953.3$ & St34 & $9.59 \pm 0.02$ & $9.40 \pm 0.02$ & $7.67 \pm 0.02$ & $5.42 \pm 0.04$ & \\
\hline 180 & $\mathrm{~J} 045510.97+302159.2$ & GMAur & $8.38 \pm 0.02$ & $8.23 \pm 0.02$ & $6.06 \pm 0.02$ & $2.52 \pm 0.02$ & \\
\hline 181 & J045545.34+301938.6 & 2 MJ04554535+3019389 & $10.16 \pm 0.02$ & $9.65 \pm 0.02$ & $8.10 \pm 0.03$ & $6.51 \pm 0.11$ & \\
\hline 182 & $\mathrm{~J} 045556.06+303620.7$ & $\mathrm{~J} 04555605+3036209$ & $8.93 \pm 0.02$ & $8.43 \pm 0.02$ & $6.04 \pm 0.02$ & $3.79 \pm 0.03$ & \\
\hline 183 & $\mathrm{~J} 045559.39+303401.4$ & SUAur & $5.07 \pm 0.05$ & $4.12 \pm 0.04$ & $2.54 \pm 0.02$ & $-0.09 \pm 0.01$ & \\
\hline 184 & $\mathrm{~J} 045601.18+302634.5$ & $\mathrm{~J} 04560118+3026348$ & $9.49 \pm 0.02$ & $9.00 \pm 0.02$ & $7.21 \pm 0.02$ & $5.91 \pm 0.05$ & \\
\hline
\end{tabular}


Table 1

Continued)

\begin{tabular}{|c|c|c|c|c|c|c|c|}
\hline $\begin{array}{l}\text { Line } \\
\text { Num. }\end{array}$ & Catalog Number & Name & $\begin{array}{c}\mathrm{W} 1(3.4 \mu \mathrm{m}) \\
\quad(\mathrm{mag})\end{array}$ & $\begin{array}{c}\mathrm{W} 2(4.6 \mu \mathrm{m}) \\
(\mathrm{mag})\end{array}$ & $\begin{array}{l}\text { W3 }(12 \mu \mathrm{m}) \\
\quad(\mathrm{mag})\end{array}$ & $\begin{array}{l}\text { W4 }(22 \mu \mathrm{m}) \\
\quad(\mathrm{mag})\end{array}$ & Notes \\
\hline 185 & J045846.27+295036.9 & None & $4.90 \pm 0.06$ & $3.60 \pm 0.04$ & $1.54 \pm 0.01$ & $0.09 \pm 0.01$ & \\
\hline 186 & J045851.41+283123.8 & BSTau & $8.60 \pm 0.02$ & $8.08 \pm 0.02$ & $6.03 \pm 0.02$ & $3.93 \pm 0.03$ & Known YSO but not traditional Taurus member \\
\hline 187 & J045903.04+300300.1 & HBHA3214-06 & $8.91 \pm 0.03$ & $8.57 \pm 0.02$ & $7.13 \pm 0.02$ & $5.14 \pm 0.03$ & Known YSO but not traditional Taurus member \\
\hline 188 & $\mathrm{~J} 050003.10+300107.4$ & GZAur & $7.70 \pm 0.02$ & $7.00 \pm 0.02$ & $5.14 \pm 0.02$ & $3.36 \pm 0.02$ & Known YSO but not traditional Taurus member \\
\hline 189 & $\mathrm{~J} 050306.59+252319.5$ & V836Tau & $8.14 \pm 0.03$ & $7.65 \pm 0.02$ & $5.89 \pm 0.02$ & $3.89 \pm 0.02$ & \\
\hline 190 & $\mathrm{~J} 050441.39+250954.4$ & CIDA-8 & $9.28 \pm 0.02$ & $8.84 \pm 0.02$ & $7.25 \pm 0.02$ & $5.20 \pm 0.03$ & \\
\hline 191 & $\mathrm{~J} 050509.00+252208.0$ & IRAS $05020+2518$ & $5.29 \pm 0.06$ & $5.11 \pm 0.03$ & $3.65 \pm 0.02$ & $2.52 \pm 0.02$ & Known YSO but not traditional Taurus member \\
\hline 192 & $\mathrm{~J} 050522.82+253130.8$ & CIDA-9 & $9.20 \pm 0.03$ & $8.31 \pm 0.02$ & $6.01 \pm 0.02$ & $4.18 \pm 0.03$ & \\
\hline 193 & $\mathrm{~J} 050623.33+243219.8$ & CIDA-11 & $9.21 \pm 0.02$ & $8.81 \pm 0.02$ & $7.13 \pm 0.02$ & $5.67 \pm 0.04$ & \\
\hline 194 & J050746.97+312018.4 & HOAur & $7.59 \pm 0.02$ & $7.22 \pm 0.02$ & $5.99 \pm 0.02$ & $4.11 \pm 0.02$ & Known YSO but not traditional Taurus member \\
\hline 195 & J050749.55+302404.9 & RWAur & $6.20 \pm 0.04$ & $5.21 \pm 0.03$ & $3.35 \pm 0.02$ & $1.48 \pm 0.02$ & \\
\hline 196 & J050754.96+250015.4 & CIDA-12 & $10.16 \pm 0.02$ & $9.79 \pm 0.02$ & $7.85 \pm 0.02$ & $5.98 \pm 0.05$ & \\
\hline
\end{tabular}

Notes.

${ }^{\text {a }}$ See the full table description in Section 3.3.

b Object identified in Rebull et al. (2010) as a candidate Taurus member, but discussed there as needing additional data to confirm/refute it as a Taurus member.

(This table is also available in a machine-readable form in the online journal.) 


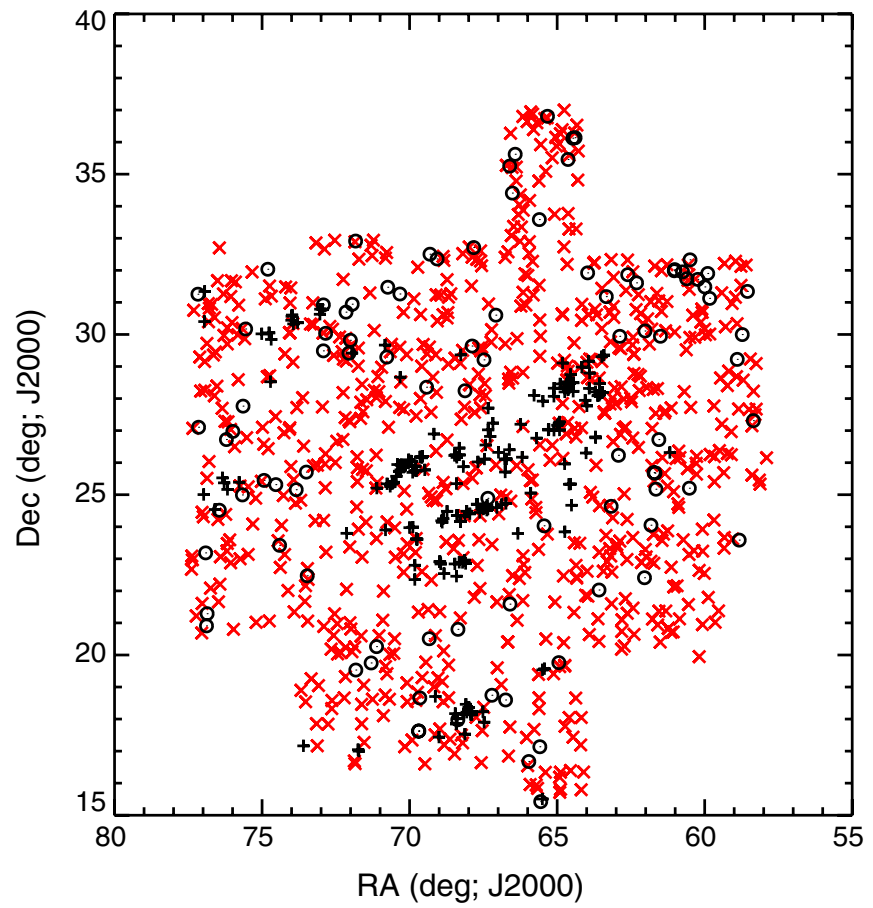

Figure 3. Location on the sky of the recovered previously identified YSOs $(+)$, the rejected objects $(x)$, and the best remaining candidates (circles). The previously identified YSOs are generally highly clustered, and the newly identified candidates are less clustered.

(A color version of this figure is available in the online journal.)

POSS, and if possible, SDSS. We then identified objects as likely subject to source contamination, resolved as a likely galaxy, or still apparently clean, relatively isolated point sources.

We have thus identified 686 objects that are confirmed or likely galaxies, 13 foreground or background stars, $1 \mathrm{PN}$, and 24 objects that seem to be subject to confusion in the relatively large WISE beam (and therefore any excess seen in the SED is likely to be contaminated by the confused source). There are 94 objects still surviving in the potential YSO candidate list. The rejected objects appear in Table 2, and the 94 surviving objects appear in Table 3. SEDs for the 94 potential YSO objects appear in the Appendix.

\section{OVERALL PROPERTIES}

We now attempt to assess the overall contamination as well as identify very high-likelihood YSOs out of our candidate list using the ancillary data we have amassed.

\subsection{Projected Location}

Figure 3 shows the location on the sky of the recovered previously identified YSOs, the rejected objects, and the surviving candidate YSOs. The previously identified YSOs are generally highly clustered along the filamentary distribution of gas and dust, and the new objects are less clustered. This is as expected, since many searches have focused on the regions already known to contain young stars, and our goal was to look for new YSOs outside the canonical groupings of previously known Taurus members. However, this could also be an indication of persistent contamination in the surviving list of YSO candidates.

Figure 4 is a more quantitative view of the clustering seen in Figure 3, showing a distribution of separations. Previously identified YSOs are very close, on average, to another previously identified YSO. The contaminants have a much broader

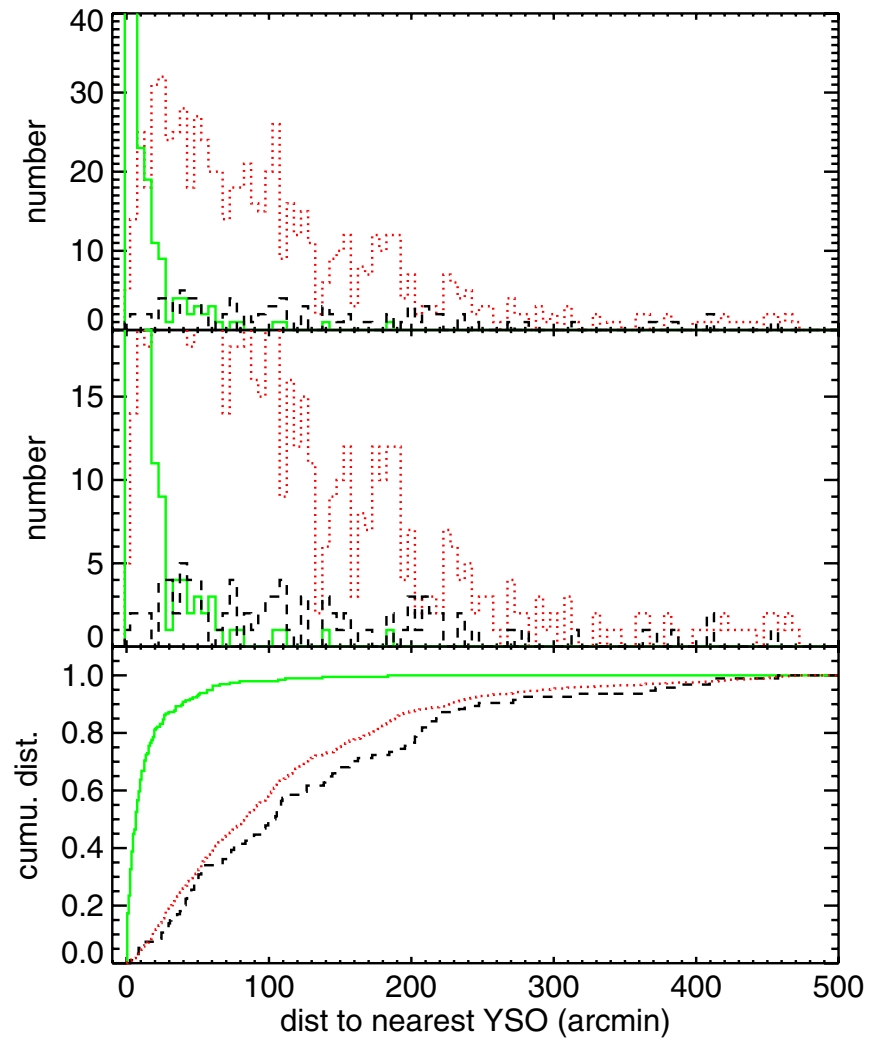

Figure 4. Histogram (top two; middle is zoomed-in version of the top) and cumulative distribution (bottom; fraction of sample with number of points less than the corresponding $x$ value) of distances in arcminutes to the nearest previously identified YSO for recovered known YSOs (green, solid line; distance is to the nearest other YSO), rejected contaminants (red, dotted line), and new YSO candidates (black, dashed line).

(A color version of this figure is available in the online journal.)

distribution and are further from the known YSOs on average. The distribution of candidate YSOs is closer to that of the contaminants than the previously identified YSOs.

In the context of proximity of objects to other known objects, as noted above, some of our objects can be found within the tidal radius of the Pleiades $\left(\sim 6^{\circ}\right.$; Adams et al. 2001). Objects identified here that are within $\sim 6^{\circ}$ of the Pleiades could belong to the Pleiades rather than Taurus. One recovered known YSO is within this radius (IRAS 04016+26102), 104 rejected contaminants, and 12 YSO candidates (J035323.82+271838.3, $\mathrm{J} 035519.09+233501.8$, J035535.05+291319.3， J040204.41+ 251210.3， J040612.75+264308.0， J040636.44+251019.1， $\mathrm{J} 040644.43+254018.1$, J040651.36+254128.3 = V1195 Tau, $\mathrm{J} 040716.73+240257.6, \quad \mathrm{~J} 040809.49+222434.8, \quad \mathrm{~J} 041141.34+$ 261341.3, and J041240.69+243815.6).

\subsection{Reddening}

Figure 5 is another look at this same issue of contamination in the candidate list, this time through the lens of $A_{V}$. The previously known YSOs are generally found in regions of high $A_{V}$, and background galaxies are found in regions of low $A_{V}$. As discussed above, we obtained a coarse estimate of $A_{V}$ based on a $\sim 6^{\prime} \times 6^{\prime}$ box centered on the position of the object in a map constructed from 2MASS star counts (Froebrich et al. 2007). Figure 5 shows that the distribution of $A_{V}$ for the candidates is considerably closer to the distribution of $A_{V}$ for the contaminants rather than the previously known YSOs. The largest $A_{V}$ found for the recovered YSOs is $10.8 \mathrm{mag}$, and the largest $A_{V}$ for 
Multiband Measurements of WISE-identified Rejected YSO Candidates in Taurus Region

\begin{tabular}{|c|c|c|c|c|c|c|c|}
\hline $\begin{array}{l}\text { Line } \\
\text { Num. }\end{array}$ & Catalog Number & Name & $\begin{array}{c}\text { W1 }(3.4 \mu \mathrm{m}) \\
(\mathrm{mag})\end{array}$ & $\begin{array}{c}\text { W2 }(4.6 \mu \mathrm{m}) \\
(\mathrm{mag})\end{array}$ & $\begin{array}{l}\text { W3 }(12 \mu \mathrm{m}) \\
(\mathrm{mag})\end{array}$ & $\begin{array}{c}\text { W4 }(22 \mu \mathrm{m}) \\
(\mathrm{mag})\end{array}$ & Notes \\
\hline 1 & J035135.81+260914.3 & 2MASX03513585+2609139 & $13.82 \pm 0.03$ & $12.94 \pm 0.03$ & $9.67 \pm 0.05$ & $7.47 \pm 0.13$ & Galaxy: extended in 2MASS, SED shape \\
\hline 2 & $\mathrm{~J} 035228.38+252002.4$ & None & $12.14 \pm 0.03$ & $10.94 \pm 0.02$ & $8.36 \pm 0.03$ & $6.34 \pm 0.06$ & Galaxy: SED shape \\
\hline 3 & J035234.58+252729.5 & None & $13.37 \pm 0.03$ & $12.28 \pm 0.02$ & $9.19 \pm 0.04$ & $6.54 \pm 0.07$ & Galaxy: SED shape \\
\hline 4 & $\mathrm{~J} 035300.20+274013.1$ & None & $13.89 \pm 0.03$ & $12.88 \pm 0.03$ & $9.99 \pm 0.06$ & $7.81 \pm 0.16$ & Galaxy: SED shape \\
\hline 5 & $\mathrm{~J} 035300.52+290542.9$ & None & $12.05 \pm 0.02$ & $11.78 \pm 0.03$ & $8.40 \pm 0.03$ & $7.03 \pm 0.08$ & Galaxy: SED shape \\
\hline 6 & J035309.58+272953.5 & 2MASX03530961+2729541 & $12.27 \pm 0.03$ & $11.11 \pm 0.02$ & $8.36 \pm 0.03$ & $6.23 \pm 0.05$ & Galaxy: extended in 2MASS, SED shape \\
\hline 7 & $\mathrm{~J} 035322.85+281358.1$ & None & $14.77 \pm 0.04$ & $13.13 \pm 0.03$ & $9.69 \pm 0.05$ & $6.96 \pm 0.08$ & Galaxy: SED shape \\
\hline 8 & J035334.67+270854.1 & None & $12.78 \pm 0.03$ & $11.67 \pm 0.02$ & $9.02 \pm 0.03$ & $6.63 \pm 0.06$ & Galaxy: SED shape \\
\hline 9 & $\mathrm{~J} 035334.93+253651.3$ & None & $11.92 \pm 0.03$ & $10.95 \pm 0.02$ & $8.18 \pm 0.03$ & $6.02 \pm 0.04$ & Galaxy: SED shape \\
\hline 10 & J035348.05+291411.3 & None & $13.52 \pm 0.03$ & $12.38 \pm 0.03$ & $9.61 \pm 0.04$ & $7.24 \pm 0.10$ & Galaxy: SED shape \\
\hline
\end{tabular}

(This table is available in its entirety in a machine-readable form in the online journal. A portion is shown here for guidance regarding its form and content.) 
Table 3

Multiband Measurements of WISE-identified New YSO Candidates in Taurus Region

\begin{tabular}{|c|c|c|c|c|c|c|c|}
\hline $\begin{array}{l}\text { Line } \\
\text { Num. }\end{array}$ & Catalog Number & Name & $\begin{array}{c}\text { W1 }(3.4 \mu \mathrm{m}) \\
(\mathrm{mag})\end{array}$ & $\begin{array}{l}\text { W2 }(4.6 \mu \mathrm{m}) \\
\quad(\mathrm{mag})\end{array}$ & $\begin{array}{l}\text { W3 }(12 \mu \mathrm{m}) \\
\quad(\mathrm{mag})\end{array}$ & $\begin{array}{l}\text { W4 }(22 \mu \mathrm{m}) \\
\quad(\mathrm{mag})\end{array}$ & Notes \\
\hline 1 & J035323.82+271838.3 & None & $11.29 \pm 0.03$ & $10.58 \pm 0.02$ & $8.66 \pm 0.03$ & $6.75 \pm 0.08$ & Within Pleiades tidal radius (Section 4.1) \\
\hline 2 & $\mathrm{~J} 035412.25+312034.0$ & None & $12.10 \pm 0.03$ & $11.76 \pm 0.02$ & $9.69 \pm 0.05$ & $7.64 \pm 0.14$ & \\
\hline 3 & J035454.27+295944.0 & None & $8.04 \pm 0.02$ & $7.34 \pm 0.02$ & $5.31 \pm 0.02$ & $4.05 \pm 0.02$ & \\
\hline 4 & J035519.09+233501.8 & None & $13.60 \pm 0.03$ & $12.79 \pm 0.03$ & $9.55 \pm 0.05$ & $6.76 \pm 0.07$ & Within Pleiades tidal radius (Section 4.1) \\
\hline 5 & J035535.05+291319.3 & None & $13.86 \pm 0.03$ & $13.06 \pm 0.03$ & $10.19 \pm 0.06$ & $7.43 \pm 0.13$ & Within Pleiades tidal radius (Section 4.1) \\
\hline 6 & J035919.63+310730.1 & None & $13.56 \pm 0.03$ & $12.39 \pm 0.03$ & $9.08 \pm 0.03$ & $6.90 \pm 0.08$ & \\
\hline 7 & J035935.02+315409.6 & 2MASX03593501+3154090 & $13.55 \pm 0.03$ & $13.12 \pm 0.03$ & $8.40 \pm 0.03$ & $4.91 \pm 0.03$ & \\
\hline 8 & J035958.81+312901.1 & HD281395 & $8.95 \pm 0.02$ & $8.95 \pm 0.02$ & $8.69 \pm 0.03$ & $6.25 \pm 0.05$ & Excess just at 22 (Section 4.6) \\
\hline 9 & J040056.13+314301.3 & None & $7.63 \pm 0.03$ & $7.14 \pm 0.02$ & $5.30 \pm 0.02$ & $2.05 \pm 0.02$ & \\
\hline 10 & J040159.15+321941.2 & HD281479 & $9.09 \pm 0.02$ & $9.09 \pm 0.02$ & $8.24 \pm 0.02$ & $6.20 \pm 0.05$ & Excess just at 22 (Section 4.6) \\
\hline 11 & J040204.41+251210.3 & None & $13.78 \pm 0.03$ & $13.04 \pm 0.03$ & $10.20 \pm 0.07$ & $7.54 \pm 0.13$ & Within Pleiades tidal radius (Section 4.1). Good JHK (Section 4.4) \\
\hline 12 & J040226.63+314409.0 & None & $11.98 \pm 0.02$ & $11.46 \pm 0.02$ & $9.45 \pm 0.04$ & $7.21 \pm 0.09$ & \\
\hline 13 & J040259.96+315703.9 & None & $9.59 \pm 0.03$ & $9.33 \pm 0.02$ & $8.44 \pm 0.03$ & $5.26 \pm 0.03$ & \\
\hline 14 & J040403.90+320116.8 & None & $13.16 \pm 0.03$ & $12.63 \pm 0.03$ & $10.18 \pm 0.07$ & $7.43 \pm 0.14$ & \\
\hline 15 & J040407.21+315914.5 & None & $12.23 \pm 0.03$ & $11.99 \pm 0.02$ & $10.13 \pm 0.06$ & $7.54 \pm 0.14$ & \\
\hline 16 & J040559.61+295638.2 & None & $7.73 \pm 0.03$ & $7.16 \pm 0.02$ & $5.22 \pm 0.02$ & $1.93 \pm 0.02$ & \\
\hline 17 & J040612.75+264308.0 & None & $14.31 \pm 0.03$ & $13.10 \pm 0.03$ & $9.39 \pm 0.04$ & $6.66 \pm 0.07$ & Within Pleiades tidal radius (Section 4.1) \\
\hline 18 & J040636.44+251019.1 & None & $14.48 \pm 0.04$ & $13.12 \pm 0.03$ & $9.34 \pm 0.04$ & $6.34 \pm 0.06$ & Within Pleiades tidal radius (Section 4.1) \\
\hline 19 & J040644.43+254018.1 & None & $11.42 \pm 0.03$ & $11.03 \pm 0.02$ & $9.25 \pm 0.04$ & $7.49 \pm 0.14$ & Within Pleiades tidal radius (Section 4.1) \\
\hline 20 & J040651.36+254128.3 & V1195Tau & $7.53 \pm 0.03$ & $7.33 \pm 0.02$ & $6.07 \pm 0.02$ & $4.56 \pm 0.02$ & Within Pleiades tidal radius (Section 4.1). Excess just at 22 (Section 4.6) \\
\hline 21 & J040716.73+240257.6 & None & $12.96 \pm 0.03$ & $11.77 \pm 0.02$ & $9.18 \pm 0.04$ & $6.91 \pm 0.09$ & Within Pleiades tidal radius (Section 4.1) \\
\hline 22 & J040805.16+300627.6 & None & $13.51 \pm 0.03$ & $12.42 \pm 0.03$ & $9.69 \pm 0.05$ & $7.34 \pm 0.12$ & Good JHK (Section 4.4) \\
\hline 23 & J040809.49+222434.8 & None & $13.63 \pm 0.03$ & $12.11 \pm 0.03$ & $9.23 \pm 0.04$ & $7.24 \pm 0.12$ & Within Pleiades tidal radius (Section 4.1) \\
\hline 24 & J040913.79+313632.5 & None & $12.15 \pm 0.02$ & $11.74 \pm 0.02$ & $10.02 \pm 0.06$ & $7.54 \pm 0.14$ & \\
\hline 25 & J041025.61+315150.7 & HD281664 & $9.88 \pm 0.03$ & $9.86 \pm 0.03$ & $9.29 \pm 0.04$ & $7.11 \pm 0.09$ & Excess just at 22 (Section 4.6) \\
\hline 26 & $\mathrm{~J} 041130.69+295628.3$ & None & $12.93 \pm 0.03$ & $11.46 \pm 0.02$ & $8.47 \pm 0.03$ & $6.05 \pm 0.05$ & \\
\hline 27 & J041141.34+261341.3 & None & $13.61 \pm 0.03$ & $12.36 \pm 0.03$ & $9.69 \pm 0.05$ & $7.34 \pm 0.12$ & Within Pleiades tidal radius (Section 4.1). Iffy $r, z$ (Section 4.5) \\
\hline 28 & J041240.69+243815.6 & None & $9.64 \pm 0.02$ & $9.24 \pm 0.02$ & $6.87 \pm 0.02$ & $4.59 \pm 0.03$ & Within Pleiades tidal radius (Section 4.1). Good $r, z$ (Section 4.5) \\
\hline 29 & J041320.01+311047.1 & HD281789 & $6.93 \pm 0.03$ & $6.38 \pm 0.02$ & $2.99 \pm 0.02$ & $0.61 \pm 0.02$ & \\
\hline 30 & J041419.20+220138.0 & None & $13.15 \pm 0.03$ & $12.22 \pm 0.03$ & $9.14 \pm 0.04$ & $6.72 \pm 0.07$ & Good JHK (Section 4.4) \\
\hline 31 & J041551.98+315514.0 & None & $12.93 \pm 0.03$ & $12.55 \pm 0.03$ & $10.28 \pm 0.08$ & $7.24 \pm 0.10$ & (SDSS spectrum: STAR -M5) good $r, z$ (Section 4.5) \\
\hline 32 & J041734.21+360814.8 & None & $12.31 \pm 0.03$ & $11.97 \pm 0.03$ & $8.45 \pm 0.03$ & $5.55 \pm 0.04$ & \\
\hline 33 & $\mathrm{~J} 041752.27+360720.2$ & None & $11.46 \pm 0.03$ & $11.45 \pm 0.03$ & $9.83 \pm 0.05$ & $7.67 \pm 0.15$ & Excess just at 22 (Section 4.6) \\
\hline 34 & J041831.61+352732.7 & None & $11.82 \pm 0.02$ & $9.47 \pm 0.02$ & $6.72 \pm 0.02$ & $3.79 \pm 0.02$ & Iffy $r, z$ (Section 4.5) \\
\hline 35 & J041946.18+194539.8 & HD284346 & $8.70 \pm 0.02$ & $8.72 \pm 0.02$ & $8.49 \pm 0.03$ & $5.84 \pm 0.04$ & Excess just at 22 (Section 4.6) \\
\hline 36 & $\mathrm{~J} 042118.39+364820.5$ & None & $14.13 \pm 0.04$ & $12.65 \pm 0.03$ & $8.86 \pm 0.03$ & $6.24 \pm 0.06$ & \\
\hline 37 & $\mathrm{~J} 042146.44+240147.1$ & None & $14.52 \pm 0.04$ & $13.16 \pm 0.04$ & $9.68 \pm 0.05$ & $6.92 \pm 0.09$ & \\
\hline 38 & J042213.75+152529.9 & None & $6.94 \pm 0.03$ & $6.39 \pm 0.02$ & $4.99 \pm 0.02$ & $1.93 \pm 0.01$ & $A_{V}>2($ Section 4.2$)$ \\
\hline 39 & $\mathrm{~J} 042220.80+170812.0$ & None & $14.05 \pm 0.03$ & $12.61 \pm 0.03$ & $9.13 \pm 0.03$ & $7.06 \pm 0.09$ & Iffy $r, z$ (Section 4.5) \\
\hline 40 & J042224.69+333459.3 & None & $11.43 \pm 0.02$ & $11.45 \pm 0.02$ & $8.84 \pm 0.03$ & $7.16 \pm 0.10$ & \\
\hline 41 & J042350.39+164035.1 & None & $14.37 \pm 0.04$ & $13.04 \pm 0.04$ & $9.46 \pm 0.04$ & $6.88 \pm 0.08$ & \\
\hline 42 & J042541.31+353718.8 & None & $8.87 \pm 0.03$ & $8.38 \pm 0.02$ & $7.27 \pm 0.02$ & $3.81 \pm 0.03$ & \\
\hline 43 & $\mathrm{~J} 042604.39+342435.7$ & None & $10.77 \pm 0.02$ & $10.35 \pm 0.02$ & $8.92 \pm 0.03$ & $6.49 \pm 0.06$ & \\
\hline
\end{tabular}


Table 3

(Continued)

\begin{tabular}{|c|c|c|c|c|c|c|c|}
\hline $\begin{array}{l}\text { Line } \\
\text { Num. }\end{array}$ & Catalog Number & Name & $\begin{array}{c}\mathrm{W} 1(3.4 \mu \mathrm{m}) \\
\quad(\mathrm{mag})\end{array}$ & $\begin{array}{c}\text { W2 }(4.6 \mu \mathrm{m}) \\
(\mathrm{mag})\end{array}$ & $\begin{array}{l}\text { W3 }(12 \mu \mathrm{m}) \\
\quad(\mathrm{mag})\end{array}$ & $\begin{array}{l}\text { W4 }(22 \mu \mathrm{m}) \\
\quad(\mathrm{mag})\end{array}$ & Notes \\
\hline 44 & J042623.36+213508.1 & None & $13.77 \pm 0.03$ & $13.02 \pm 0.05$ & $10.03 \pm 0.09$ & $6.65 \pm 0.11$ & \\
\hline 45 & J042625.87+351507.6 & None & $10.44 \pm 0.03$ & $9.56 \pm 0.02$ & $7.36 \pm 0.02$ & $5.53 \pm 0.04$ & Good $r, z$ (Section 4.5) \\
\hline 46 & J042659.19+183548.7 & None & $12.98 \pm 0.03$ & $11.81 \pm 0.02$ & $8.57 \pm 0.03$ & $5.77 \pm 0.04$ & Iffy $r, z$ (Section 4.5) \\
\hline 47 & $\mathrm{~J} 042818.51+303605.3$ & None & $14.40 \pm 0.04$ & $13.62 \pm 0.05$ & $9.20 \pm 0.04$ & $7.10 \pm 0.10$ & \\
\hline 48 & J042850.54+184436.1 & None & $9.79 \pm 0.03$ & $9.42 \pm 0.03$ & $7.19 \pm 0.02$ & $5.09 \pm 0.04$ & $A_{V}>2($ Section 4.2$)$ \\
\hline 49 & J042923.42+245314.8 & None & $11.94 \pm 0.03$ & $11.68 \pm 0.03$ & $9.22 \pm 0.04$ & $6.97 \pm 0.08$ & \\
\hline 50 & J042954.77+291228.5 & None & $10.61 \pm 0.02$ & $10.56 \pm 0.02$ & $9.66 \pm 0.05$ & $6.74 \pm 0.08$ & Excess just at 22 (Section 4.6) \\
\hline 51 & J043118.56+324237.8 & None & $13.81 \pm 0.03$ & $13.03 \pm 0.04$ & $9.92 \pm 0.06$ & $7.43 \pm 0.13$ & \\
\hline 52 & J043131.61+293819.0 & None & $13.73 \pm 0.03$ & $12.96 \pm 0.03$ & $9.96 \pm 0.06$ & $7.17 \pm 0.09$ & \\
\hline 53 & J043229.33+281434.9 & None & $13.91 \pm 0.03$ & $13.01 \pm 0.04$ & $9.50 \pm 0.04$ & $6.74 \pm 0.08$ & \\
\hline 54 & J043326.74+204758.7 & None & $12.76 \pm 0.03$ & $11.56 \pm 0.03$ & $9.06 \pm 0.04$ & $6.91 \pm 0.08$ & Iffy $r, z$ (Section 4.5) \\
\hline 55 & J043327.90+175843.8 & None & $10.05 \pm 0.02$ & $8.88 \pm 0.02$ & $6.53 \pm 0.02$ & $4.07 \pm 0.03$ & Good JHK (Section 4.4) \\
\hline 56 & J043616.13+322119.4 & None & $11.64 \pm 0.02$ & $11.51 \pm 0.02$ & $9.71 \pm 0.05$ & $6.15 \pm 0.05$ & \\
\hline 57 & J043714.47+323011.7 & None & $14.28 \pm 0.04$ & $13.63 \pm 0.05$ & $9.10 \pm 0.03$ & $6.05 \pm 0.05$ & \\
\hline 58 & J043720.50+203024.4 & None & $11.69 \pm 0.03$ & $11.64 \pm 0.02$ & $9.45 \pm 0.04$ & $6.95 \pm 0.08$ & \\
\hline 59 & J043741.83+282112.6 & None & $14.26 \pm 0.03$ & $13.20 \pm 0.03$ & $9.37 \pm 0.04$ & $6.54 \pm 0.06$ & \\
\hline 60 & J043837.06+183934.2 & None & $14.05 \pm 0.03$ & $12.91 \pm 0.03$ & $9.73 \pm 0.05$ & $6.53 \pm 0.06$ & \\
\hline 61 & J043845.02+173743.1 & None & $10.30 \pm 0.02$ & $9.88 \pm 0.02$ & $8.07 \pm 0.02$ & $6.02 \pm 0.05$ & \\
\hline 62 & $\mathrm{~J} 043847.26+173725.9$ & None & $11.39 \pm 0.02$ & $10.90 \pm 0.02$ & $9.04 \pm 0.03$ & $7.05 \pm 0.09$ & \\
\hline 63 & $\mathrm{~J} 044118.60+311544.2$ & None & $12.27 \pm 0.03$ & $11.41 \pm 0.03$ & $8.55 \pm 0.03$ & $6.75 \pm 0.08$ & \\
\hline 64 & J044257.69+312822.6 & None & $12.67 \pm 0.03$ & $11.39 \pm 0.02$ & $8.18 \pm 0.02$ & $5.98 \pm 0.05$ & \\
\hline 65 & J044305.62+291737.6 & None & $13.66 \pm 0.03$ & $13.18 \pm 0.03$ & $10.98 \pm 0.12$ & $7.38 \pm 0.12$ & \\
\hline 66 & J044428.98+201537.5 & None & $13.16 \pm 0.03$ & $12.62 \pm 0.03$ & $7.96 \pm 0.02$ & $4.89 \pm 0.03$ & Iffy $r, z$ (Section 4.5$)$ \\
\hline 67 & J044512.68+194501.1 & None & $13.54 \pm 0.03$ & $12.51 \pm 0.03$ & $9.30 \pm 0.04$ & $6.94 \pm 0.08$ & Good JHK (Section 4.4) \\
\hline 68 & J044717.49+193149.1 & None & $14.11 \pm 0.03$ & $12.99 \pm 0.03$ & $9.65 \pm 0.05$ & $6.80 \pm 0.07$ & \\
\hline 69 & J044719.33+325449.8 & None & $13.58 \pm 0.03$ & $12.35 \pm 0.03$ & $9.33 \pm 0.04$ & $6.72 \pm 0.07$ & Good $J H K$ (Section 4.4) \\
\hline 70 & $\mathrm{~J} 044746.77+305635.8$ & 2MASX04474674+3056355 & $11.53 \pm 0.03$ & $11.06 \pm 0.03$ & $9.22 \pm 0.04$ & $7.06 \pm 0.10$ & \\
\hline 71 & J044801.88+294902.1 & 2MASX04480189+2949023 & $13.49 \pm 0.03$ & $12.69 \pm 0.03$ & $9.62 \pm 0.05$ & $7.13 \pm 0.10$ & Good JHK (Section 4.4) \\
\hline 72 & J044813.48+292453.5 & None & $8.44 \pm 0.02$ & $7.74 \pm 0.02$ & $5.33 \pm 0.02$ & $3.53 \pm 0.02$ & Good JHK (Section 4.4) \\
\hline 73 & J044837.31+304145.1 & None & $12.29 \pm 0.03$ & $12.28 \pm 0.03$ & $10.07 \pm 0.06$ & $7.16 \pm 0.09$ & \\
\hline 74 & J045122.95+300206.8 & None & $11.98 \pm 0.02$ & $11.78 \pm 0.02$ & $9.03 \pm 0.03$ & $6.91 \pm 0.09$ & \\
\hline 75 & J045141.49+305519.5 & 2MASX04514147+3055196 & $12.36 \pm 0.02$ & $11.73 \pm 0.02$ & $7.90 \pm 0.02$ & $5.20 \pm 0.04$ & $A_{V}>2($ Section 4.2$)$ \\
\hline 76 & J045141.56+292918.9 & None & $14.58 \pm 0.04$ & $13.23 \pm 0.04$ & $9.60 \pm 0.05$ & $6.82 \pm 0.08$ & \\
\hline 77 & $\mathrm{~J} 045352.77+222813.7$ & None & $12.07 \pm 0.03$ & $10.97 \pm 0.02$ & $8.07 \pm 0.03$ & $5.67 \pm 0.04$ & Good $J H K$ (Section 4.4) \\
\hline 78 & $\mathrm{~J} 045400.09+254219.2$ & None & $13.96 \pm 0.03$ & $13.22 \pm 0.03$ & $9.94 \pm 0.06$ & $7.04 \pm 0.09$ & \\
\hline 79 & J045522.17+250923.2 & None & $13.43 \pm 0.03$ & $12.25 \pm 0.03$ & $9.02 \pm 0.03$ & $6.45 \pm 0.06$ & Iffy $r, z$ (Section 4.5) \\
\hline 80 & J045737.44+232513.9 & None & $10.99 \pm 0.02$ & $10.98 \pm 0.02$ & $8.71 \pm 0.03$ & $5.89 \pm 0.04$ & \\
\hline 81 & J045808.02+251852.6 & None & $10.54 \pm 0.02$ & $10.59 \pm 0.02$ & $9.88 \pm 0.06$ & $7.40 \pm 0.12$ & Good $r, z$ (Section 4.5). Excess just at 22 (Section 4.6) \\
\hline 82 & J045913.28+320201.3 & None & $11.99 \pm 0.02$ & $10.83 \pm 0.02$ & $8.03 \pm 0.02$ & $5.47 \pm 0.04$ & Good $J H K$ (Section 4.4) \\
\hline
\end{tabular}


Table 3

Continued)

\begin{tabular}{|c|c|c|c|c|c|c|c|}
\hline $\begin{array}{l}\text { Line } \\
\text { Num. }\end{array}$ & Catalog Number & Name & $\begin{array}{l}\text { W1 }(3.4 \mu \mathrm{m}) \\
\quad(\mathrm{mag})\end{array}$ & $\begin{array}{l}\text { W2 }(4.6 \mu \mathrm{m}) \\
\quad(\mathrm{mag})\end{array}$ & $\begin{array}{l}\text { W3 }(12 \mu \mathrm{m}) \\
\quad(\mathrm{mag})\end{array}$ & $\begin{array}{c}\text { W4 }(22 \mu \mathrm{m}) \\
\quad(\mathrm{mag})\end{array}$ & Notes \\
\hline 83 & $\mathrm{~J} 045943.63+252647.2$ & None & $14.58 \pm 0.04$ & $12.99 \pm 0.03$ & $9.36 \pm 0.04$ & $6.93 \pm 0.09$ & Iffy $r, z$ (Section 4.5) \\
\hline 84 & $\mathrm{~J} 050213.63+301014.2$ & None & $13.63 \pm 0.03$ & $12.38 \pm 0.03$ & $9.47 \pm 0.04$ & $7.19 \pm 0.10$ & \\
\hline 85 & J050234.82+274549.6 & None & $10.77 \pm 0.02$ & $10.36 \pm 0.02$ & $7.57 \pm 0.02$ & $5.37 \pm 0.04$ & \\
\hline 86 & J050239.85+245933.5 & None & $10.31 \pm 0.03$ & $9.76 \pm 0.02$ & $7.38 \pm 0.02$ & $6.13 \pm 0.05$ & \\
\hline 87 & $\mathrm{~J} 050357.98+265828.8$ & HD284075 & $9.27 \pm 0.02$ & $9.27 \pm 0.02$ & $8.87 \pm 0.03$ & $6.09 \pm 0.05$ & Excess just at 22 (Section 4.6) \\
\hline 88 & $\mathrm{~J} 050450.13+264314.6$ & HD32509 & $6.92 \pm 0.03$ & $6.94 \pm 0.02$ & $5.81 \pm 0.02$ & $3.35 \pm 0.02$ & Excess just at 22 (Section 4.6) \\
\hline 89 & J050547.69+243058.9 & None & $11.66 \pm 0.03$ & $11.53 \pm 0.02$ & $8.74 \pm 0.03$ & $7.35 \pm 0.11$ & \\
\hline 90 & J050726.91+211716.5 & None & $13.36 \pm 0.03$ & $12.60 \pm 0.03$ & $9.43 \pm 0.04$ & $6.98 \pm 0.09$ & \\
\hline 91 & $\mathrm{~J} 050731.86+205428.2$ & None & $12.06 \pm 0.02$ & $12.07 \pm 0.02$ & $10.32 \pm 0.07$ & $7.74 \pm 0.15$ & \\
\hline 92 & $\mathrm{~J} 050739.03+231106.5$ & None & $11.00 \pm 0.03$ & $10.64 \pm 0.02$ & $9.16 \pm 0.04$ & $7.48 \pm 0.13$ & \\
\hline 93 & $\mathrm{~J} 050836.48+270614.7$ & None & $11.89 \pm 0.03$ & $11.41 \pm 0.02$ & $9.16 \pm 0.04$ & $7.31 \pm 0.12$ & \\
\hline 94 & $\mathrm{~J} 050841.75+311521.9$ & None & $11.08 \pm 0.02$ & $10.59 \pm 0.02$ & $9.00 \pm 0.03$ & $7.41 \pm 0.12$ & \\
\hline
\end{tabular}

(This table is also available in a machine-readable form in the online journal.) 


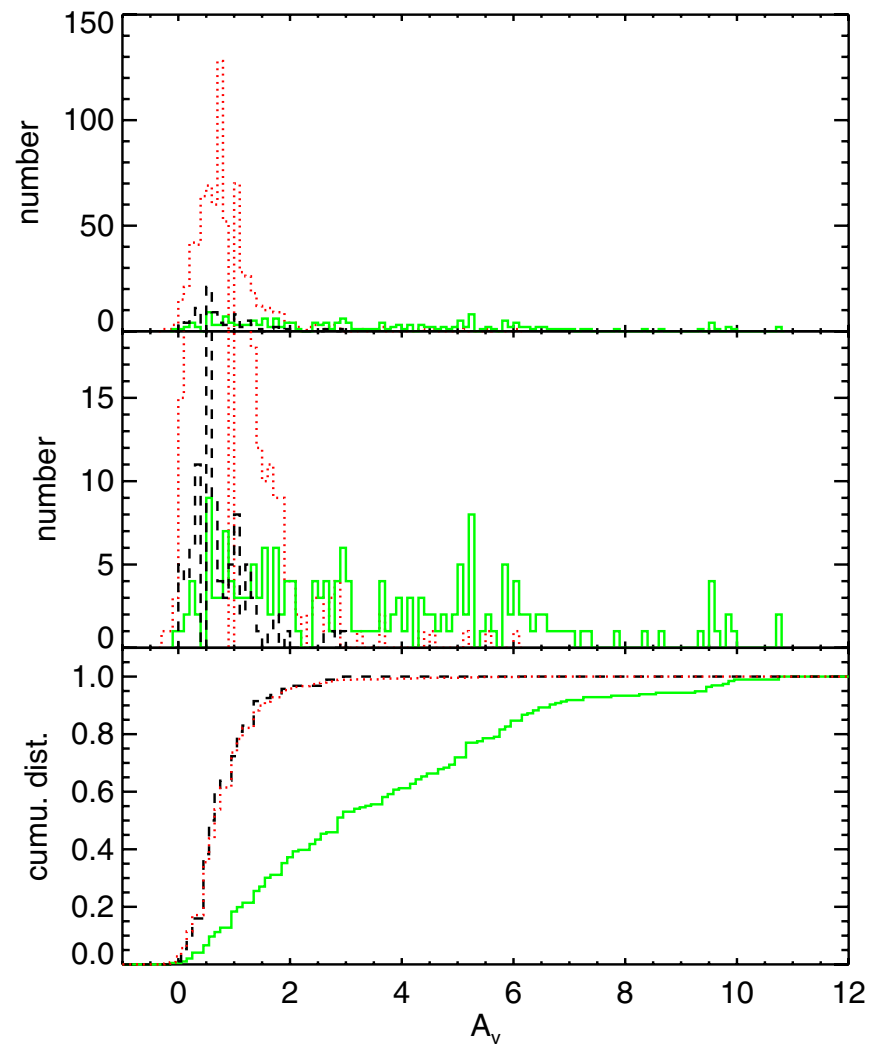

Figure 5. Histogram of $A_{V}$ values (top two; middle is zoomed-in version of the top), and cumulative distribution of $A_{V}$ values (bottom), for recovered known YSOs (green, solid line), rejected contaminants (red, dotted line), and new YSO candidates (black, dashed line). The distribution of $A_{V}$ values for the candidates is much closer to the distribution of contaminants than previously known YSOs. The $A_{V}$ estimates come from a median $\sim 6^{\prime} \times 6^{\prime}$ box centered on the position of the object.

(A color version of this figure is available in the online journal.)

the candidates is only $3 \mathrm{mag}$ (J042850.54+184436.1), with two others having $A_{V}>2\left(\mathrm{~J} 042213.75+152529.9-A_{V}=2.6\right.$, $\left.\mathrm{J} 045141.49+305519.5-A_{V}=2.7\right)$. These may be among the highest likelihood YSOs on our candidate list. This analysis could indicate that our list of surviving candidate YSOs is substantially contaminated, or it could reflect instead the fact that all the searches to date (including our prior Spitzer survey) have focused on regions of high $A_{V}$, and the "discovery space" is in the regions of lower $A_{V}$.

Figure 6 shows the distribution of recovered YSOs, new YSO candidates, and rejected YSO candidates on the $A_{V}$ map from Froebrich et al. (2007). As inferred above, the rejected objects tend to be evenly distributed, and the recovered YSOs tend to be clustered in regions of high $A_{V}$. The new objects are not particularly clustered, but not evenly distributed either. There is an apparent clump of objects in the northwest, toward Perseus (see also Figure 2); most of these objects have excesses only at $22 \mu \mathrm{m}$, which, as discussed below, may be a result of source confusion.

\subsection{W1 Brightnesses}

The color cuts in Koenig et al. (2011) are performed in, among other color spaces, W1 versus W1-W4 ([3.4] versus [3.4]-[22]). This diagram, and its Spitzer analog ([3.6] versus [3.6]-[24]), has proven to be very useful in identifying YSO candidates (see, e.g., Rebull et al. 2010). Figure 7 shows this diagram for the 94 YSO candidates, rejected candidates, and recovered YSOs. Photospheres (stars without disks) would have a $\mathrm{W} 1-\mathrm{W} 4$ color near zero, and galaxies near $\mathrm{W} 1 \sim 16$, $\mathrm{W} 1-\mathrm{W} 4 \sim 7$, but they have already been removed by the Koenig et al. (2011) process. Most of the previously identified YSOs are bright, and most of the contaminants are faint. The new YSO candidates span the range of bright and faint.

The distribution of brightnesses at W1 $(3.4 \mu \mathrm{m})$ can be seen explicitly in Figure 8. Figure 8 suggests a different conclusion than Figure 5; here, the new candidates are intermediate in cumulative brightness distribution between the previously known YSOs (brighter) and the contaminants (fainter). This assessment is reinforced by the formal values calculated for two-sided two-dimensional Kolmogorov-Smirnov tests. This is consistent with broad expectations; most of the background galaxies will be faint, most of the surveys to date will have found the bright YSOs, and the "discovery space" for new YSOs is on the faint end of the distribution of expected brightnesses for YSOs in Taurus. Unextincted YSOs in Taurus above the hydrogen burning limit should be no fainter than 13th magnitude at W1. Thus, fainter YSOs should show evidence of extinction, and much fainter ones could only be edge-on disks or more distant emission-line objects.

Figure 8 suggests that our YSO list is contaminated to a larger degree at the faint end of the distribution of $3.4 \mu \mathrm{m}$ magnitudes. There is a small peak in the distribution of candidate magnitudes at the same location of the highest peak of contaminant magnitudes (W1 $\sim 13.5 \mathrm{mag}$ ), suggesting that the highest fraction of contaminants per bin will be found in those bins. Similar results are obtained for, e.g., a histogram of $H$ magnitudes. However, it is worth noting that in our earlier studies of Taurus, we found a large number of bright background stars (e.g., AGBs) that appeared to have excesses, but are not members of Taurus.

\subsection{Near-IR Colors}

Figure 9 shows the $J H K_{s}$ color-color diagram for the 94 YSO candidates, rejected candidates, and recovered known YSOs. Consistent with expectations and with Figure 5 above, the distribution of the previously identified YSOs extends from the locus of normal young and accreting stars with near-infrared excesses in the direction of the reddening vector. Encouragingly, the distribution of contaminants is broad and generally consistent with non-stellar sources. Few of the YSO candidates have large values of $A_{V}$, consistent with Figure 5. There are 11 objects with $\left(H-K_{s}\right)>0.75$ and $(J-H)>1$ : J040204.41+251210.3, J040805.16+300627.6, J041419.20+220138.0， J043131.61+293819.0， J043327.90+ 175843.8， J044512.68+194501.1， J044719.33+325449.8, J044801.88+294902.1， J044813.48+292453.5， J045352.77+ 222813.7, and J045913.28+320201.3. These objects are in the right regime of the $J H K_{s}$ diagram to be higher-quality YSO candidates. However, as can be seen in the Appendix, we have no ancillary photometric data for most of these, and for the two where we do have such data, the WISE measurements do not agree with those from Akari (J044813.48+292453.5) or IRAC $(\mathrm{J} 043131.61+293819.0=0431316+293818$ in our Spitzer catalog). This is not generally the case-usually the WISE, Akari, and Spitzer measurements are in good agreement. One reason they might not agree would be the differing resolution of the instruments-if there is source confusion or the source is resolved (e.g., it is a likely galaxy), then the photometry would not agree. However, these objects are point sources in all of the images we examined. Another reason why the photometry might be different is that the young stars themselves may vary between 

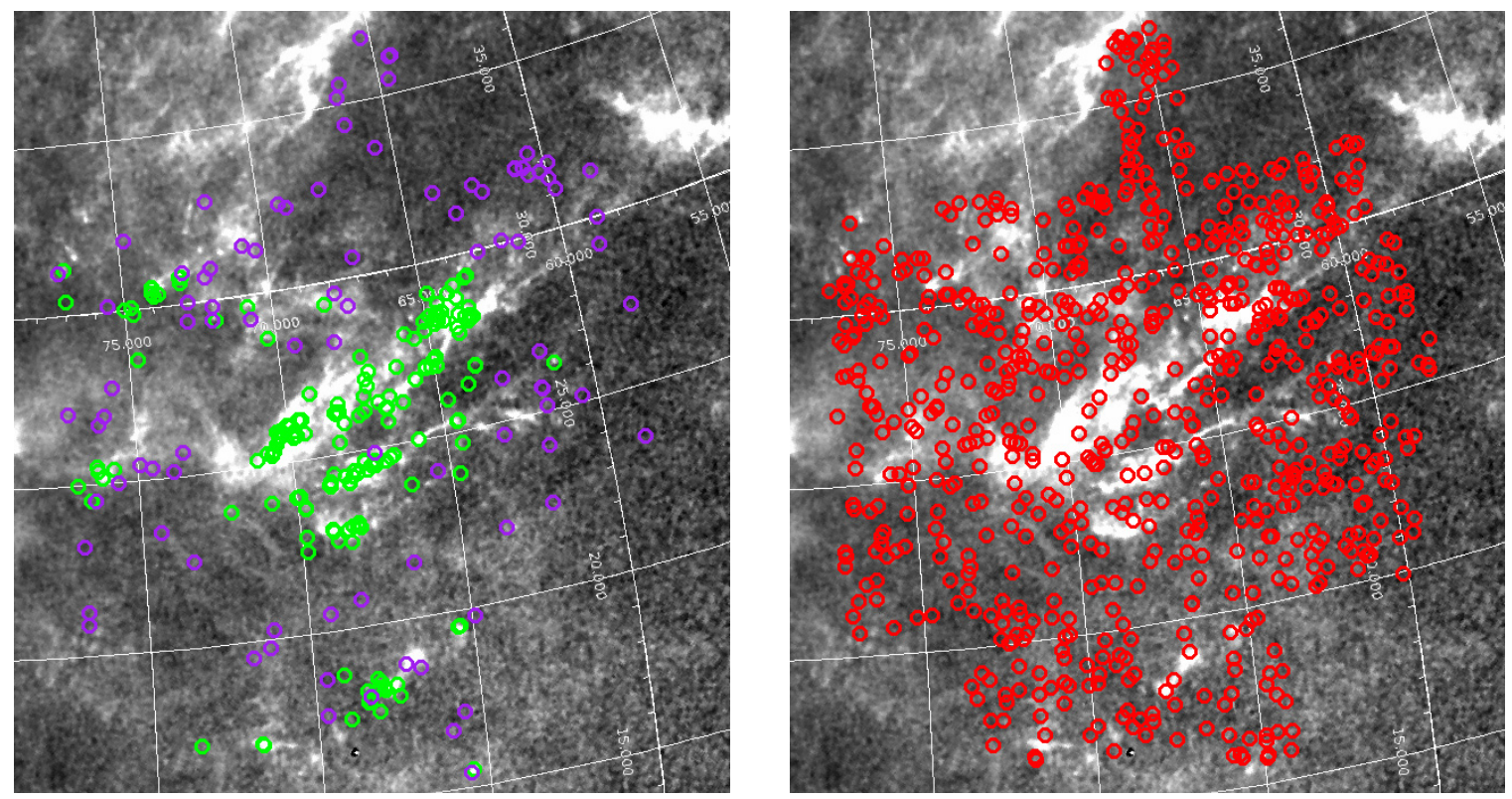

Figure 6. Locations of YSO candidates and recovered previously identified YSOs (left, purple and green, respectively) and rejected candidates (right) superimposed on an $A_{V}$ map constructed from 2MASS by Froebrich et al. (2007) with $\sim 4^{\prime}$ resolution. The structure seen in the $A_{V}$ can be compared to that seen in $I R A S 100 \mu \mathrm{m}$ in Figure 2.

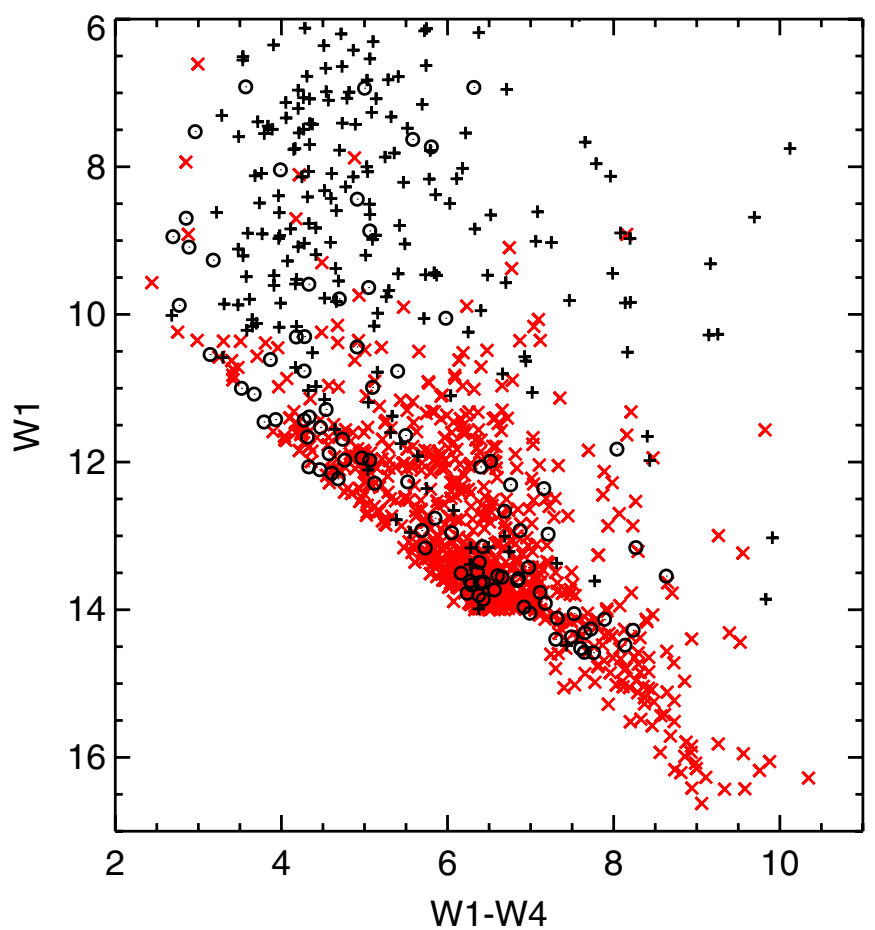

Figure 7. W1 vs. W1-W4 ([3.4] vs. [3.4]-[22]) color-magnitude diagram for the recovered previously identified YSOs $(+)$, the rejected candidates $(x)$, and the best remaining YSO candidates (circles).

epochs (see, e.g., Morales-Calderon et al. 2011, or Rebull 2011, and references therein). Additional observations are warranted.

\subsection{Optical Data}

The SDSS data do not extend over our entire studied region, but optical data can be very helpful in ruling out YSO candidates. There are matches to SDSS spectra for only 27 objects in our entire set, 15 of which are tagged galaxies (including known YSOs CoKu Tau/1 and GV Tau; presumably the SDSS pipeline

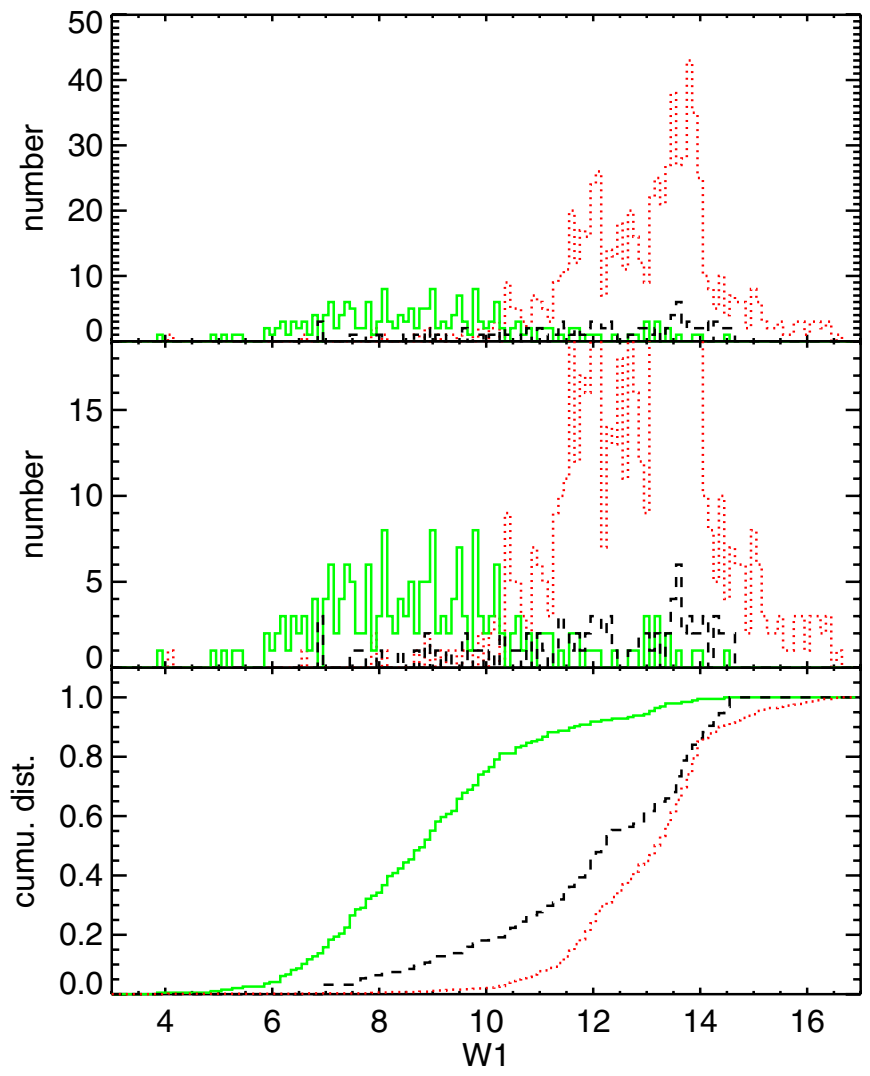

Figure 8. Histogram of W1 (3.4 $\mu$ m values; top two, where middle is zoomedin version of the top), and cumulative distribution of $\mathrm{W} 1$ values (bottom), for recovered known YSOs (green, solid line), rejected contaminants (red, dotted line), and new YSO candidates (black, dashed line). Unlike the $A_{V}$ distributions in the previous figure, here the distribution of values for the candidates much more similar to that for the previously known YSOs than that for the contaminants.

is confused by emission lines in these sources). Eleven more of the SDSS spectra are for previously identified YSOs. Thirteen of the objects that we have placed in our "reject" list have SDSS 


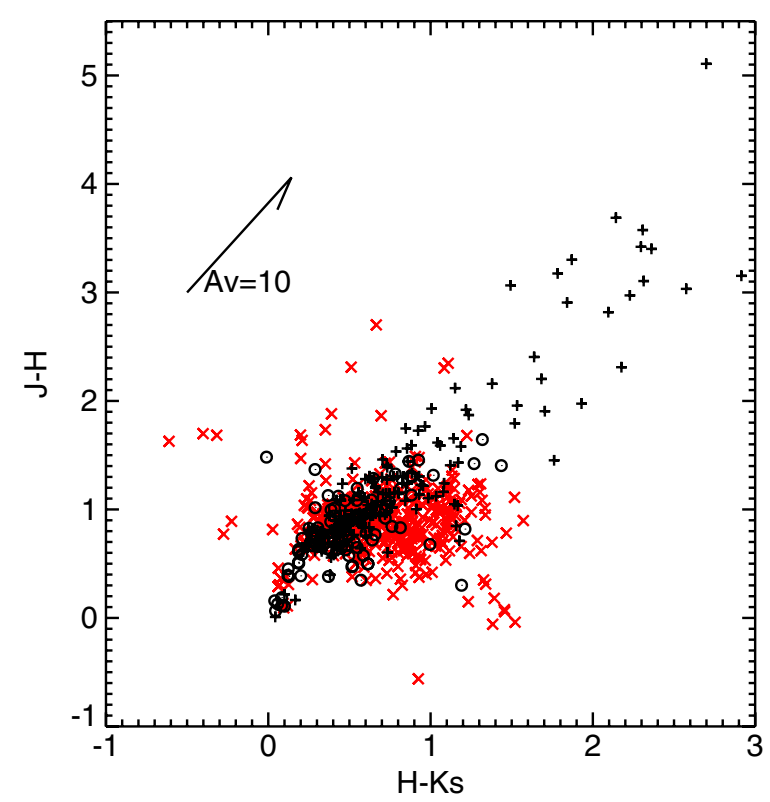

Figure 9. $J H K_{s}$ color-color diagram for the recovered previously identified YSOs (+), the rejected candidates $(x)$, and the best remaining YSO candidates (circles).

(A color version of this figure is available in the online journal.)

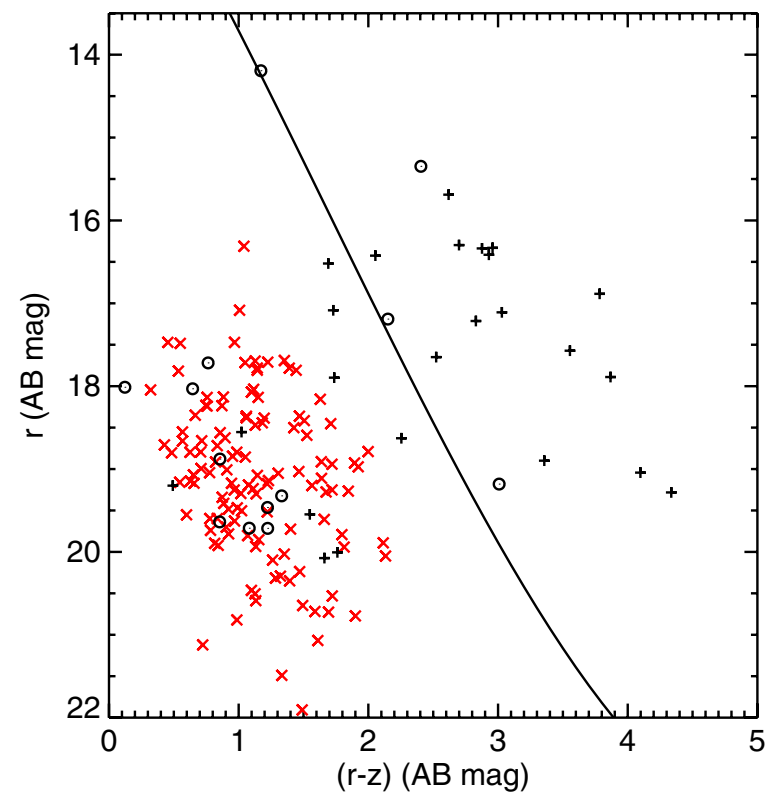

Figure 10. SDSS $r$ vs. $(r-z)$ in AB magnitudes for the recovered previously identified YSOs $(+)$, the rejected candidates $(x)$, and the best remaining candidates (circles). The main sequence from Bochanski et al. (2010) is the solid line. Legitimate Taurus objects could be below the main sequence as a result of edge on disks; in those cases, we are seeing scattered light from substantial infrared excesses viewed at a high inclination angle.

(A color version of this figure is available in the online journal.)

spectra, all of which are galaxies. Just one of our surviving YSO candidates has an SDSS spectrum, and it is identified as an M5. Since our placement of objects on the reject or candidate YSO list was independent of the SDSS spectra, this is encouraging and suggests that our classification of the remaining objects without SDSS spectra may be correct on average.

There are SDSS $z$ measurements for $\sim 26 \%$ of our entire set, but only $\sim 15 \%$ of our surviving YSO candidate list. Figure 10 shows the $r$ versus $(r-z)$ color-magnitude diagram for our

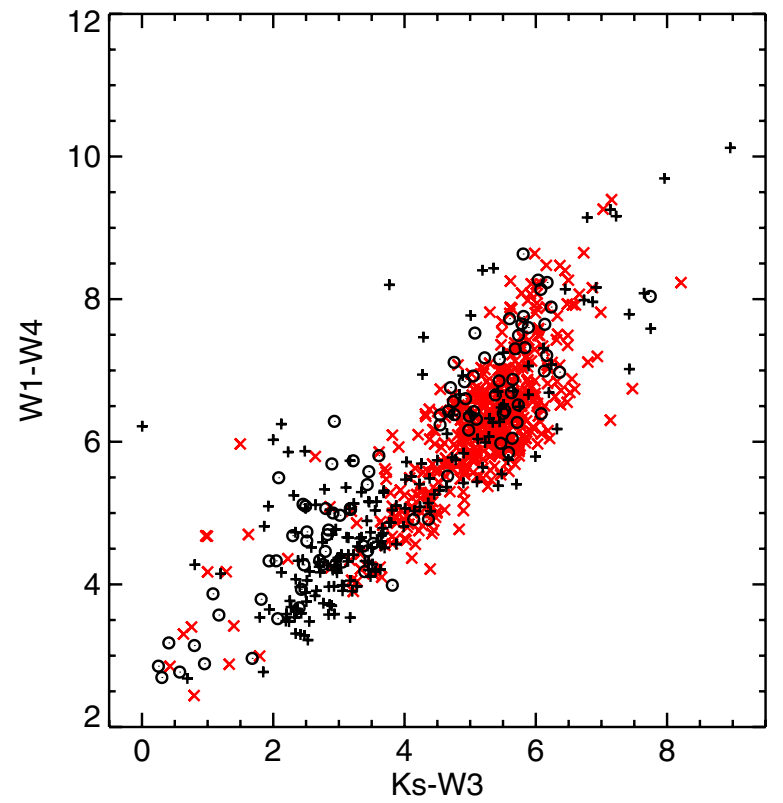

Figure 11. W1-W4 ([3.4]-[22]) vs. $K_{\mathrm{s}}-\mathrm{W} 3\left(K_{\mathrm{s}}-[12]\right)$ for the recovered previously identified YSOs $(+)$, the rejected candidates $(\times)$, and the best remaining candidates (circles).

(A color version of this figure is available in the online journal.)

objects. Most of the recovered known YSOs are in the expected location, e.g., to the red side in the diagram. Just four of our candidate YSOs are in the main portion of the distribution of known YSOs (J041240.69+243815.6, J041551.98+315514.0, $\mathrm{J} 042625.87+351507.6$, and J045808.02+251852.6). It is worth noting that the distribution of previously identified YSOs as well as candidates extends into the regime occupied by the contaminants on the blue side, but that all five of the previously identified YSOs in that area $(r<1.9$ and $(r-z)<1.9)$ are actually unconfirmed YSOs from Rebull et al. (2010) (J041604.83+261800.9, J041810.60+284447.0, $\mathrm{J} 041823.20+251928.0$, J041858.99+255740.0, and J041940.48 +270100.7), and may in the end turn out not to be Taurus members. The nine new YSO candidates in that regime may have a similar fate; they are J041141.34+261341.3, J041831.61+352732.7， J042146.44+240147.1， J042220.80+ 170812.0, J042659.19+183548.7, J043326.74+204758.7, $\mathrm{J} 044428.98+201537.5, \mathrm{~J} 045522.17+250923.2$, and J045943.63+ 252647.2.

\subsection{Size of Infrared Excess}

Since the WISE spatial resolution is relatively low, especially if there is an infrared excess seen only at $22 \mu \mathrm{m}$, one must be suspicious of source confusion. The long wavelength excess objects may also be the most interesting astrophysically, since they could have large inner disk holes. We have investigated each of the images and have not been able to identify source confusion. Objects that we have identified that have excesses only at $22 \mu \mathrm{m}$ can be selected out of Figure 11. Objects with small $K_{\mathrm{s}}-\mathrm{W} 3$ excesses $(<2)$ generally also have small $\mathrm{W} 1-\mathrm{W} 4$ excesses, but the eleven that also have $\mathrm{W} 1-\mathrm{W} 4<4$ are the ones for which we are most suspicious of source confusion: J035958.81+312901.1, J040159.15+321941.2， J040651.36+254128.3， J041025.61+ $315150.7, \quad \mathrm{~J} 041752.27+360720.2, \quad \mathrm{~J} 041946.18+194539.8$, J042954.77+291228.5， J045808.02+251852.6， J050357.98+ 265828.8, and J050450.13+264314.6. 
Table 4

Comparison of Gutermuth et al. (2008, 2009) and Koenig et al. (2011) Methods

\begin{tabular}{lccc}
\hline \hline Koenig et al. Classification & $\begin{array}{c}\text { Gutermuth et al. } \\
\text { Contaminant }\end{array}$ & $\begin{array}{c}\text { Gutermuth et al. } \\
\text { Class 0/I }\end{array}$ & $\begin{array}{c}\text { Gutermuth et al. } \\
\text { Class II }\end{array}$ \\
\hline Class I & 2 & 65 & 19 \\
Class II & 5 & 17 & 84 \\
Transition disk & 12 & 2 & 4 \\
\hline
\end{tabular}

Table 5

Breakdown of the Koenig et al. (2011) Classifications

\begin{tabular}{lccc}
\hline \hline Sample & Class I & Class II & Transition Disk \\
\hline Of the rejects (number) & 365 & 211 & 148 \\
Of the rejects (fraction) & 0.50 & 0.29 & 0.20 \\
Of the recovered (number) & 62 & 122 & 12 \\
Of the recovered (fraction) & 0.32 & 0.62 & 0.06 \\
Of the candidates (number) & 28 & 46 & 20 \\
Of the candidates (fraction) & 0.29 & 0.49 & 0.21 \\
\hline
\end{tabular}

\subsection{Distribution of SED Classifications}

In Rebull et al. (2010), we compared a variety of YSO selection methods, and we also had enough ancillary data that we were able to categorize most of the objects that WISE sees in this overlap region; Figure 3 shows relatively few WISE-identified new YSO candidates in the region where we had Spitzer data (compare to Figure 1). In the region of Spitzer coverage, we can compare the selection statistics for the Koenig et al. (2011) method with that from Gutermuth et al. (2008, 2009). There are IRAC-1 measurements for $\sim 25 \%$ of our set. There are 210 for which we can calculate a Gutermuth et al. classification, 119 of which are recovered known YSOs. Both the Koenig et al. and Gutermuth et al. methods return SED classifications of objects. Table 4 shows a comparison of the methods. For the most part, the classifications agree-Gutermuth et al. identify relatively few of the sources as contaminants $(9 \%)$, and both methods agree that $31 \%$ of the sample are Class I and $40 \%$ are Class II.

Finally, we consider, among the objects we have recovered, rejected, or retained as YSO candidates, the distribution of classifications returned by the Koenig et al. (2011) method; see Table 5. The rejected sources are predominantly categorized as Class I candidates, consistent with the observation that many have very flat SEDs suggestive of extragalactic objects (or a reflection of our selection bias against very flat SEDs). The recovered YSOs are mostly categorized as Class II, consistent with what is already known in Taurus. The set of surviving YSO candidates is also mostly categorized as Class II objects.

\section{CONCLUSIONS}

We have presented the results of our search for new candidate Taurus members using 2MASS+WISE data over $\sim 260 \mathrm{deg}^{2}$. We used NIR+MIR colors to select a set of YSO candidates following methodology presented by Koenig et al. (2011). We compared the resultant list of YSO candidates to our own catalog from Rebull et al. (2010), updated to include Taurus members not in our original Spitzer map, the 2MASS Extended Source Catalog, several SDSS stripes (photometry plus spectroscopy), and Akari data. We recover 196 previously identified young stars in the direction of Taurus with infrared excess and identify 686 objects that are confirmed or likely galaxies, 13 foreground or background stars, 1 PN, 24 objects likely subject to confusion based on inspection of the images, and 94 new YSO candidates. As was the case for our smaller Spitzer-based search for YSO candidates (Rebull et al. 2010), supporting ancillary data and manual inspection of the images and SEDs for each YSO candidate are critical. The YSO candidates are broadly distributed in (projected) space, unlike the previously identified YSOs, which are generally clustered. Few are in regions of high $A_{V}$ (again, unlike the recovered YSO sample), but they are generally fainter in $3.4 \mu \mathrm{m}$ than the previously identified sample. There is likely to be contamination lingering in this list. We identify a few objects that are particularly likely to be legitimate YSOs, and a few that are particularly likely to be contaminants. Follow-up spectra to obtain spectral types and to search for emission lines and other indications of youth are warranted.

This publication makes use of data products from the Widefield Infrared Survey Explorer, which is a joint project of the University of California, Los Angeles, and the Jet Propulsion Laboratory/California Institute of Technology, funded by the National Aeronautics and Space Administration. This research has made use of NASA's Astrophysics Data System (ADS) Abstract Service, and of the SIMBAD database, operated at CDS, Strasbourg, France. This research has made use of data products from the Two Micron All Sky Survey (2MASS), which is a joint project of the University of Massachusetts and the Infrared Processing and Analysis Center, funded by the National Aeronautics and Space Administration and the National Science Foundation. These data were served by the NASA/IPAC Infrared Science Archive, which is operated by the Jet Propulsion Laboratory, California Institute of Technology, under contract with the National Aeronautics and Space Administration. This research has made use of the Digitized Sky Surveys, which were produced at the Space Telescope Science Institute under U.S. Government grant NAG W-2166. The images of these surveys are based on photographic data obtained using the Oschin Schmidt Telescope on Palomar Mountain and the UK Schmidt Telescope. The plates were processed into the present compressed digital form with the permission of these institutions.

The research described in this paper was partially carried out at the Jet Propulsion Laboratory, California Institute of Technology, under contract with the National Aeronautics and Space Administration.

\section{APPENDIX}

\section{SEDs FOR ALL OF THE CANDIDATES}

For each of our new candidate Taurus members, we provide an SED here in Figure 12. Notation is as follows: "+"-literature Johnson photometry, "**-Sloan photometry, " $\times$ "-CFHT photometry, diamonds-2MASS, circles-IRAC, stars-WISE, triangles-Akari, and squares-MIPS. Limits for any band, if available, are indicated by arrows. The wavelength is in 


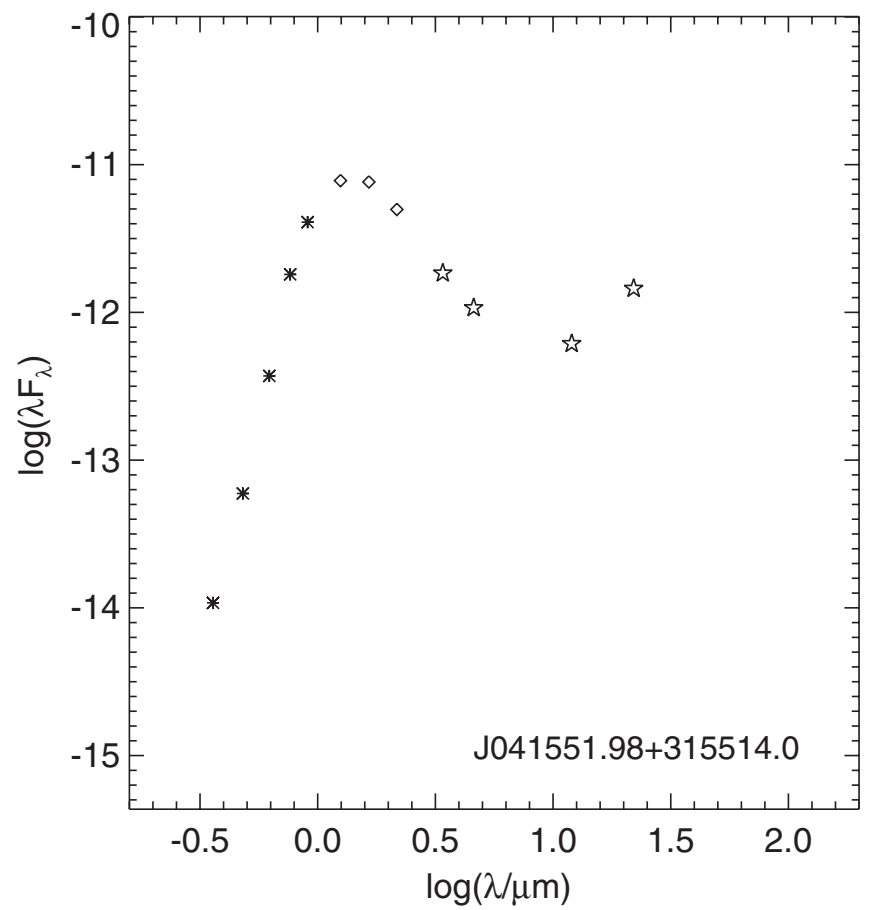

Figure 12. SEDs for the new candidate members, shown here for J041551.98+315514. Notation is as follows: "+"-literature Johnson photometry, " $*$ "-Sloan photometry, " $\times$ "-CFHT photometry, diamonds-2MASS, circles-IRAC, stars-WISE, triangles-Akari, squares-MIPS. Limits for any band, if available, are indicated by arrows. The wavelength is in microns; $\lambda F_{\lambda}$ is in cgs units ( $\mathrm{erg} \mathrm{s}^{-1} \mathrm{~cm}^{-2}$ ). Each plot has the WISE catalog number, which can also be found in Table 3 .

(The complete figure set (94 images) is available in the online journal.)

microns; $\lambda F_{\lambda}$ is in cgs units ( $\mathrm{erg} \mathrm{s}^{-1} \mathrm{~cm}^{-2}$ ). Each plot has the WISE catalog number, which can also be found in Table 3 .

Note added in proof: E. Mamajek, via private communication, notes that Tycho-2 and UCAC3 proper motions for several of the objects do not seem to have a proper motion consistent with the Taurus subgroups. The motions of HD 281395, 281479, $281664,281789,284346$, and 284075 are all very small-they are probably background objects. HD 32509 is a previously known Ae star with IR excess-it seems to be an isolated young massive star with a disk, but its proper motion (pmRA, pmDec
$=+15,-32$ mas $\left.\mathrm{yr}^{-1}\right)$ isn't similar to that of the group that it is nearest to (L1544; pmRA, pmDec $=+0.9,-17.6$ mas $\mathrm{yr}^{-1}$; Table 8 of Luhman et al. 2009). The clump near RA, Dec $\sim 60$, +31 (HD 281395, 281479, 281664, 281789) might be members of Per OB2 association at $d \sim 300$ pc.

\section{REFERENCES}

Adams, J., Stauffer, J., Monet, D., Skrutskie, M., \& Beichman, C. 2001, AJ, 121,2053

Allen, L. E., Calvet, N., D’Alessio, P., et al. 2004, ApJS, 154, 363

Bochanski, J., Hawley, S. L., Covey, K. R., et al. 2010, AJ, 139, 2679

Finkbeiner, D., Padmanabhan, N., Schlegel, D. J., et al. 2004, AJ, 128, 2577

Froebrich, D., Murphy, G., Smith, M., Walsh, J., \& Del Burgo, C. 2007, MNRAS, 378, 1447

Goldsmith, P., Heyer, M., Narayanan, G., et al. 2008, ApJ, 680, 428

Güdel, M., Briggs, K. R., Arzner, K., et al. 2007a, A\&A, 468, 353

Güdel, M., Padgett, D., \& Dougados, C. 2007b, in Protostars and Planets V, ed. B. Reipurth, D. Jewitt, \& K. Keil (Tucson, AZ: Univ. Arizona Press), 329

Gutermuth, R., Megeath, S. T., Myers, P. C., et al. 2009, ApJS, 184, 18

Gutermuth, R. A., Myers, P. C., Megeath, S. T., et al. 2008, ApJ, 674, 336

Harvey, P., Mern, B., Huard, T. L., et al. 2007, ApJ, 663, 1149

Herbig, G. H., Andrews, S., \& Dahm, S. 2004, AJ, 128, 1233

Hernandez, J., Hartmann, L., Calvet, N., et al. 2008, ApJ, 686, 1195

Ishihara, D., Onaka, T., Kataza, H., et al. 2010, A\&A, 514, A1

Jarrett, T., et al. 2011, ApJ, submitted

Kenyon, S., Gómez, M., \& Whitney, B. A. 2008, in the Handbook of Star Forming Regions, Vol. I, ed. B. Reipurth (The Northern Sky ASP Monograph Publications, Vol. 4; San Francisco, CA: ASP), 405

Koenig, X., et al. 2011, ApJ, submitted

Luhman, K., Allen, P. R., Espaillat, C., Hartmann, L., \& Calvet, N. 2010, ApJS, 186,111

Luhman, K. L., Mamajek, E. E., Allen, P. R., \& Cruz, K. L. 2009, ApJ, 703, 399

Morales-Calderon, M., Stauffer, J. R., Hillenbrand, L. A., et al. 2011, ApJ, 733, 50

Padgett, D. L., Rebull, L. M., Stapelfeldt, K. R., et al. 2008a, ApJ, 672, 1013

Padgett, D., et al. 2008b, Taurus Legacy Delivery Document (available at http://ssc.spitzer.caltech.edu/legacy/taurushistory.html)

Padmanabhan, N., Schlegel, D. J., Finkbeiner, D. P., et al. 2008, ApJ, 674, 1217

Rebull, L. 2011, in Proc. Cool Stars, Stellar Systems, and the Sun 16 (arXiv:1101.5647)

Rebull, L., Guieu, S., Stauffer, J. R., et al. 2011, ApJS, 193, 25

Rebull, L., Padgett, D. L., McCabe, C.-E., et al. 2010, ApJS, 186, 259

Rebull, L. M., Stapelfeldt, K. R., Evans, N. J., II, et al. 2007, ApJS, 171, 447

Skrutskie, M., Cutri, R. M., Stiening, R., et al. 2006, AJ, 131, 1163

Slesnick, C., Carpenter, J. M., Hillenbrand, L. A., \& Mamajek, E. E. 2006, AJ, 132,2665

Werner, M., Roellig, T. L., Low, F. J., et al. 2004, ApJS, 154, 1

Wright, E., Eisenhardt, P. R. M., Mainzer, A. K., et al. 2010, AJ, 140, 1868 